\title{
Phosphorylation of the transcription factor Atf1 at multiple sites by the MAP kinase Sty1 controls homologous recombination and transcription
}

Laura Sánchez-Mir†, Rodrigo Frailè, José Ayté and Elena Hidalgo

Oxidative Stress and Cell Cycle Group, Universitat Pompeu Fabra, Barcelona, Spain

Correspondence to Elena Hidalgo: elena.hidalgo@upf.edu (EH)

tL.S.-M. and R.F. contributed equally to this work.

Running Title: Activation of Atf1 by phosphorylation at multiple sites 


\section{ABSTRACT}

Transcription factors are often the downstream effectors of signaling cascades. In fission yeast, the transcription factor Atf1 is phosphorylated by the MAP kinase Sty1 under several environmental stressors to promote transcription initiation of stress genes. However, Sty1 and Atf1 have also been involved in other cellular processes such as homologous recombination at hotspots, ste11 gene expression during mating and meiosis, or regulation of $f b p 1$ gene transcription under glucose starvation conditions. Using different phospho-mutants of Atf1, we have investigated the role of Atf1 phosphorylation by Sty1 in those biological processes. An Atf1 mutant lacking the canonical MAP kinase phosphorylation sites cannot activate $f b p 1$ transcription when glucose is depleted, but it is still able to induce recombination at ade6.M26 and to induce ste11 after nitrogen depletion; in these last cases, Sty 1 is still required, suggesting that additional non-canonical sites are activating the transcription factor. In all cases, an Atf1 phosphomimetic mutant bypasses the requirement of the Sty1 kinase in these diverse biological processes, highlighting the essential role of the DNA binding factor Atf1 on chromatin remodeling and cell adaptation to nutritional changes. We propose that posttranslational modifications of Atf1 by Sty1, either at canonical or non-canonical sites, are sufficient to activate some of the functions of Atf1, those involving chromatin remodelling and transcription initiation. However, in the case of $f b p 1$ where Atf1 acts synergistically with other transcription factors, elimination of the canonical sites is sufficient to hamper some of the interactions required in this complex scenario and to impair transcription initiation.

Keywords: transcription regulation; Schizosaccharomyces pombe; Sty1; Atf1; homologous recombination; phosphorylation; fbp1; ste11

\section{Abbreviations used:}

TF, Transcription Factor; MAP kinase, Mitogen-Activated Protein Kinase; S/TP, serine/threonineproline; bZIP, basic zipper; Pol II, RNA polymerase II; CRE, cAMP-response element; STRE, StressResponse Element; UAS, Upstream Activation Sequence; mlonRNA, metabolic long non coding RNA; TSS, transcriptional start site; ChIP, chromatin immuno-precipitation; MNase, Micrococcal nuclease; SAGA, Spt-Ada-Gcn5 acetyltransferase; SD, standard deviation. 


\section{INTRODUCTION}

The response to environmental cues in all eukaryotic organisms is triggered by mitogen-activated protein MAP kinase pathways that up-regulate stress-dependent gene expression programs to allow cell adaptation [1]. After stress imposition, phosphorylation of MAP kinase promotes its translocation to the nucleus and triggers phosphorylation of transcription factors (TFs). Activated TFs adapt the complex RNA polymerase II (Pol II) transcriptional machinery into particular sets of genes to mediate specific changes in the gene expression program in order to turn extracellular signals into specific cellular responses [2].

The fission yeast Schizosaccharomyces pombe responds to environmental stressors through the Sty1/Spc1 MAP kinase pathway, which is activated by phosphorylation events in response to several stress conditions and elicits a wide change of the gene expression program at the transcriptional level [3-7]. Upon stress, Sty1 is phosphorylated and accumulates in the nucleus to promote transcriptional activation or repression of genes [7-10], and these transcriptional changes are totally or partially dependent on the Atf1 TF $[6,11]$. Atf1 is a basic zipper (bZIP)-containing TF that form heterodimers with Pcr1, another bZIP protein. Upon stress, this TF is phosphorylated by Sty1, and it is worth to mention that nuclear accumulation of the kinase is not sufficient to induce phosphorylation of Atf1: Sty1 phosphorylation is also required to activate its kinase activity and phosphorylate the TF $[12,13]$.

In response to extracellular hydrogen peroxide $\left(\mathrm{H}_{2} \mathrm{O}_{2}\right)$ at sub-lethal concentration, more than 500 genes are up-regulated more than 2-fold in a Sty1-Atf1 dependent manner $[5,6]$. The role of the signal-induced phosphorylation of Atf1 by Sty 1 in transcription activation was not clear: it had been proposed to be relevant only as a protein stabilization factor [14]. However, we recently described that a phosphomimetic Atf1 mutant is able to engage the anti-stress transcriptional program in a Sty1independent manner. Phosphorylation of the TF per se does not largely contribute to Atf1 recruitment to DNA, but rather promotes transcription initiation. The main function of Sty1 in transcription activation is to promote Atf1 phosphorylation, since expression of phosphomimetic Atf1 mutants bypasses the requirement of the MAP kinase [11]. Interestingly, cells expressing Atf1 mutants lacking the canonical MAP kinase phospho sites (serine/threonine-proline S/TP sites) are sensitive to oxidative stress due to the lack of transcriptional induction of a subset of stress genes, including ctt1 
and $\operatorname{sr} x 1$ [11]; these genes are co-synergistically regulated by Atf1 and another TF, Pap1. On the contrary, genes such as gpd 1 and $h s p 9$, only dependent on active Atf1, are not largely affected by expression of a hypo-phosphorylation mutant of Atf1, even though this induction is still dependent on the Sty 1 kinase $[11,15]$. We proposed that phosphorylation of Atf1 by Sty1 establishes a platform for interactions with the basal transcriptional machinery (either Pol II itself, chromatin remodelers or other TFs) to facilitate transcription initiation [11].

Apart from their essential role in the activation of gene transcription under stress conditions, Sty1-Atf1-Pcr1 have also been implicated in other cellular processes such as meiotic homologous recombination at the mutated chromosomal locus ade6-M26. The binding of the bZIP-containing proteins to this hotspot is due to a single base pair substitution at the M26 allele of the ade6 gene, which generates a cAMP-response element (CRE)-like DNA site 5'-ATGACGT-3' [16]. TF binding promotes meiotic recombination at this particular hotspot [and at other naturally occurring Atf1 binding sites [17, 18]] through formation of double-strand DNA breaks around it by topoisomerase II-like protein Rec12/Spo11 [19], although this process seems to be independent of Atf1 phosphorylation [20].

The Sty1-Atf1 pathway has also an important role in the response to nutrient starvation, with nitrogen or glucose deprivation triggering the activation of the cascade. Sty1 is involved in the initiation of the mating and meiosis program through regulation of ste11 levels, a master regulator of this process [21-23]. Accumulation of the ste11 transcripts upon nitrogen depletion is triggered by activation of the Sty1-Atf1-Pcr1 pathway and inactivation of the cAMP-dependent Pka1 kinase by Cgs1 [9, 24]. When Pka1 is inactivated, it can no longer phosphorylate the TF Rst2, substrate of Pka1, which becomes active after its hyper-phosphorylation via other kinases, and suffers a cytoplasm-to-nucleus translocation [25]. However, CRE sites are not present at the ste11 promoter, and neither Atf1, nor Pcr1 or Sty1 have been found at this gene in a genome-wide study [26]. Indeed, up-regulation of ste11 by the Sty1-Atf1 pathway is an indirect process: Sty1-Atf1 regulates cgs2 transcription; it codes for the phosphodiesterase Cgs2, a negative regulator of the cAMP-dependent protein kinase (protein kinase A) pathway [27].

Regarding glucose starvation, it has been described that Sty1 and Atf1 are also involved in the response to glucose starvation by transcriptional regulation of the fructose-1,6-bisphosphatase coding gene (fbp1), a key enzyme in the gluconeogenic pathway [28]. Upon glucose depletion, Atf1 and 
Rst2 TFs participate in the progressive modification of chromatin configuration in the fbp1 promoter region to an open state $[29,30]$. The binding sites for Atf1 and Rst2 at the fbp 1 promoter region have been named upstream activation sequences UAS1 and UAS2, respectively [31], and are more than 600 base pairs apart. UAS1 contains the conserved CRE site to which Atf1 has high affinity, while UAS2 contains the stress-response element (STRE site) which is the target sequence for Rst2 [31]. In the current model, the chromatin of fbp 1 promoter region remains in a closed configuration. When glucose is depleted, Atf1 is recruited at UAS1 site promoting the transcription of a cascade of metabolic long non coding RNAs (mlonRNAs) that induce the opening of chromatin around UAS1 and UAS2 [32, 33]. Likewise, mlonRNAs transcription also facilitates promoter association of Atf1, enhancing fbp 1 gene expression [34]. The binding of Atf1 to UAS1 is stabilized by Rst2, probably through the formation of a chromatin loop which spatially brings both UAS close together [30]. UAS2 site also serves as a binding site for another $\mathrm{C}_{2} \mathrm{H}_{2} \mathrm{Zn}$ finger TF, the Scr1 repressor. Rst2 and Scr1 reciprocally regulate the induction of $f b p 1$ in low glucose conditions [35]. Thus, chromatin immunoprecipitation assays revealed a switching of Scr1 to Rst2 bound at UAS2 during glucose derepression. The high glucose-dependent repressive role of Scr1 has been characterized in depth in the genes coding for the high-affinity glucose transporter Ght5 [36] and for the TF Rsv1 [37]. After glucose starvation, Scr1 is quickly exported from the nucleus to the cytoplasm, at which time Rst2 is imported into the nucleus.

In all the above scenarios, Atf1-dependent transcription or recombination initiation has been associated with chromatin remodeling [27, 38, 39], but only in the case of fbp 1 additional TFs participate in the process. We aimed at deciphering the role of the signal-dependent phosphorylation of Atf1 by Sty 1 in the previously described biological processes. To this end, we have expressed HAtagged wild-type Atf1 from a constitutive promoter, as well as hypo-phosphorylated and phosphomimetic mutants, and studied their behavior in homologous recombination and in the activation of the ste11 and fbp 1 genes upon nitrogen or glucose depletion, respectively. We demonstrate that Atf1 phosphorylation is not required for the regulation of recombination at ade6.M26 and the induction of ste11 after nitrogen starvation, but it is critical to activate fbp 1 gene transcription when glucose is depleted. Importantly, Atf1 phosphomimetic mutant bypasses the requirement of the Sty1 kinase in all those biological processes, revealing the essential role of the Atf1 TF and its phosphorylation status on chromatin remodeling and cell adaptation to stress. 


\section{RESULTS}

\section{Atf1 phospho-mutants and chronological aging}

We designed and constitutively expressed two types of Atf1 phosphorylation mutants: HA-Atf1.10M has ten out of eleven putative MAP kinase phosphorylation sites mutated to alanine or isoleucine, and therefore is a hypo-phosphorylation mutant, while the HA-Atf1.10D, with substitutions to acidic residues, is a phosphomimetic one (Figure $1(\mathrm{a})$ ). The $11^{\text {th }}$ SP site at position $438-439$ lies close to the bZIP domain and has been shown before to have no impact on Atf1 activity as a TF [11]. These mutants allowed us to identify two subsets of stress genes, so that cells expressing HA-Atf1.10M are unable to activate one group (i.e. ctt1 or $s r \times 1$ ) but can still activate the other one in a Sty1-dependent manner (Figure 1(b)). Expression of HA-Atf1.10D is sufficient to trigger the expression of both sets of genes in a Sty1-independent manner, either in response to peroxides (ctt1, srx1) or constitutively (gpd1, hsp9).

Sty1 and Atf1 were previously reported to participate in the regulation of lifespan [40, 41]. Thus, cells lacking Sty1 display reduced viability during stationary phase, while strain $\Delta p y p 1$, encoding a negative regulator of the cascade, is a long-lived mutant [40]. As shown in Figure 1(c) and previously published [40], glucose-rich cultures of wild-type and $\Delta$ sty 1 cells are not viable five days after reaching stationary phase. Similar to what has been reported for $\Delta p y p 1$ [40], cells expressing HA-Atf1.10D are long-lived, even in the absence of Sty1 (Figure 1(c)).

\section{Cells expressing HA-Atf1.10M are still able to activate ste11 after nitrogen starvation}

Sty 1 and Atf1 are also involved in the transcriptional activation of the ste 11 or $f b p 1$ genes, and they also trigger meiotic recombination (Figure $1(\mathrm{~d}))[17,18,21-23,28]$. We first confirmed that $\Delta$ atf1 cells expressing HA-Atf1 display a wild type pattern of activation of ste11 and its downstream gene, mei2 [22] (Figure S1A). As indicated in the Introduction, Sty1 and Atf1 do not regulate ste11 directly in response to nitrogen depletion, but rather activate transcription of the cgs2 gene, coding for a negative regulator of the Pka1 pathway [27]. We confirmed that ste11 and mei2 transcription is totally abolished in $\Delta c g s 2$ cells expressing any of the HA-Atf1 mutant derivatives, even HA-Atf1.10D (Figure S1B). In order to study the role of Atf1 phosphorylation in the up-regulation of the cgs2-ste11-mei2 cascade (Figure 2(a)), we expressed HA-Atf1 and its mutant derivatives in $\Delta$ atf1 cells, and measured 
cgs2, ste11 and mei2 transcripts upon nitrogen starvation by quantitative PCR. As shown in Figure 2(bcd), expression of either HA-Atf1 or its two mutant derivatives are capable of activating these genes. However, only cells expressing HA-Atf1.10D were able to induce ste11 (Figure 2(e)) or mei2 (Figure S1C) transcription after nitrogen starvation in cells lacking Sty1.

Because ste11 induction and cell cycle G1 phase arrest are both essential to initiate the mating and meiosis program [42], we also studied the role of Atf1 phosphorylation in the G1 phase arrest after nitrogen depletion. As described before [42], 75\% of wild-type cells accumulate in G1 16 hours after nitrogen starvation according to flow cytometry analysis (Figure 2(f), left panel), while G1 arrest is significantly deficient in cells lacking Atf1. Expression of HA-Atf1 or both mutant derivatives can suppress this effect. Again, only HA-Atf1.10D phosphomimetic mutant is able to bypass the absence of Sty1 (Figure 2(f), bottom right panel).

In conclusion, upon nitrogen starvation both Atf1 and Sty1 are required for ste11 induction and G1 arrest [42], and HA-Atf1.10M mutant is able to complement the lack of Atf1 but not the absence of Sty1. This suggests that other non-canonical MAP kinase phosphorylation sites in Atf1 are required for full activation of the TF at either the cgs2 or the ste11 promoters, as described before for the stress genes gpd1 and hsp9 [11]. As reported for these genes, expression of the phosphomimetic mutant HA-Atf1.10D is able to activate ste11 in the absence of Sty1. In an attempt to genetically trace those additional phosphorylation sites, we first confirmed that the Atf1.11M mutant, lacking the last MAP kinase site in Atf1, is still capable of inducing ste11 and mei2 (Figure S2A). We then introduced four and six mutations, based on phospho-proteomic and genetic reports [42-44] (our unpublished results), to generate the HA-Atf1.15M and HA-Atf1.17M, but both are still able to promote ste11 and mei2 transcription (Figure S2B).

\section{HA-Atf1.10M is able to induce recombination at the ade6-M26 hotspot, but only when Sty1 is present}

To determine the role of Atf1 phosphorylation in homologous recombination at ade6-M26, we determined whether the hotspot is active by measuring the frequency of meiotic recombination between cells expressing the different Atf1 phospho-mutants (HA-Atf1, HA-Atf1.10M and HAAtf1.10D) and harboring two sets of ade6 alleles (Figure 3(a)). The ade6-M26 allele contains a DNA binding site for the Atf1-Pcr1 heterodimer, whereas the ade6-M375 and ade6-M210 alleles do not 
(Figure 3(b)). As reported before [20], crosses between strains harboring the ade6-M375 and ade6M210 alleles revealed basal (control) recombination levels, while crosses between strains harboring the ade6-M26 and ade6-M210 alleles displayed elevated recombination levels, which depend on the presence of Atf1.

In $\Delta$ atf1 cells expressing wild-type HA-Atf1, crosses harboring ade6-M26 allele displayed a frequency of ade6+ recombinants significantly higher than crosses harboring the control (M375) allele, which demonstrates the activation of hotspot (Figure 3(c)) [18]. The frequency of recombinants from control (M375) crosses in $\Delta$ atf1 and cells expressing wild-type Atf1 protein mutants was similar. However, M26 DNA site-dependent recombination increase was abolished in $\Delta$ atf1 mutant. So, Atf1 is not required to maintain basal recombination but is essential to activate the ade6-M26 meiotic recombination hotspot [45]. Figure 3(c) shows similar levels of recombination at the hotspot in $\Delta$ atf1 cells expressing HA-Atf1 and both phospho-mutants, demonstrating that HA-Atf1.10M is sufficient to induce meiotic homologous recombination at ade6-M26 hotspot, as previously shown [20]. Nevertheless, when we delete Sty1, levels of ade6-M26 recombination in HA-Atf1 and HA-Atf1.10M mutants were drastically reduced to basal levels (M375), while HA-Atf1.10D retains its recombination activity (Figure 3(c)). These results suggest that both Atf1 and Sty1 are required to trigger homologous recombination at the ade6-M26 hotspot [17], and probably Sty1 can phosphorylate Atf1 at non-canonical sites to promote its activation as a recombination factor.

In response to low glucose, Atf1 phosphorylation is essential for the recruitment of Atf1 to the fbp1 promoter

Sty1 and Atf1 also respond to glucose deprivation to trigger transcription of genes such as $f b p 1$ (Figure 4(a)) [28]. Figure 4(b) illustrates the fbp1 promoter based on numerous reports (see Introduction). The binding sites for Atf1 (UAS1) and Rst2 (UAS2) relative to the translation origin (+1) are indicated, although both TFs can be detected in both sites [30]. The relative nucleosome positions, extracted from a genome-wide study [46], are also indicated, with arbitrary numbers -1 to -7 starting upstream of the transcriptional start site (TSS). To study the role of Atf1 phosphorylation in the response to glucose starvation, we analyzed fbp 1 transcription in cells expressing the Atf1 phospho-mutants. Northern blot analysis revealed that neither $\Delta$ atf1 mutant nor cells expressing the hypo-phosphorylation mutant HA-Atf1.10M are able to trigger fbp 1 transcription 1 hour after a shift to 
low glucose medium (Figure 4(c), upper panel), while cells expressing the phosphomimetic HAAtf1.10D show similar levels of fbp1 transcripts than wild type cells (Figure 4(c) upper panel). Again, expression of the phosphomimetic HA-Atf1.10D bypasses the requirement of Sty1 in the transcriptional response to glucose depletion (Figure 4(c) lower panel).

In order to check whether Atf1 phosphorylation affects its recruitment to the fbp1 promoter, we performed chromatin immuno-precipitation (ChIP) analysis. We analyzed the binding of Atf1 at UAS1 and UAS2 sites in glucose rich conditions and after different time points of glucose starvation. As shown in Figure 4(d), HA-Atf1 and HA-Atf1.10D are recruited at both UAS sites after glucose depletion, while the HA-Atf1.10M binding is largely suppressed. Therefore, Atf1 phosphorylation is important for its recruitment to the fbp 1 promoter after glucose deprivation.

Since Rst2 recruitment to the fbp1 promoter region in glucose starved cells is Atf1-dependent [47], the next step was to analyze Rst2 binding at UAS1 and UAS2 sites in cells expressing HA-Atf1 and mutant derivatives. In cells lacking Atf1 or expressing HA-Atf1.10M we did not detect Rst2-FLAG recruitment at UAS1 and UAS2 sites after glucose starvation, while HA-Atf1 and Atf1.10D promoted its binding at both sites (Figure 4(e)). In cells lacking Rst2, both recruitment to DNA of HA-Atf1, HAAtf1.10M and HA-Atf1.10D (Figure 4(f)) and fbp1 transcription (Figure 4(g)) upon glucose depletion are fully abolished. Therefore, the HA-Atf1.10D is not able to overcome the absence of Rst2 in promoting fbp 1 transcription; similarly, it cannot promote $c t t 1$ and $s r x 1$ expression in cells lacking Pap1 [11]. In conclusion, these results demonstrate that Atf1 has to be phosphorylated by Sty1 in order to ensure the recruitment of both Atf1 and Rst2 TFs to the fbp1 promoter.

\section{Nucleosome eviction from the fbp1 promoter after glucose starvation is dependent on Atf1 phosphorylation}

Given the importance of Atf1 phosphorylation for its binding to the fbp1 promoter after glucose starvation, and taking into account that the TF is essential to promote chromatin remodeling in this region [47], we decided to study the effect of the Atf1 phospho-mutants in nucleosome organization at the fbp 1 promoter by nucleosome scanning. Briefly, we treated chromatin from cells expressing wild type HA-Atf1, $\triangle$ atf1 and HA-Atf1.10M cells with micrococcal nuclease (MNase), isolated mononucleosomes and PCR-amplified them with pairs of overlapping primers encompassing more than $1 \mathrm{~kb}$, from -100 to -1250 base pairs relative to the translation initiation site, as described 
elsewhere [48]. The nucleosome pattern obtained in HA-Atf1-expressing cells in basal conditions (Figure 5(a)) was very similar to the previously described genome-wide analysis for wild-type cells [46], with nucleosomes stably positioned in a region spanning the whole fbp 1 promoter.

Nucleosomes $-1,-2,-4$ and -6 are fully evicted in cells expressing HA-Atf1 1 hour after glucose starvation (Figure 5(a)), while nucleosomes -3 and -5 are partially maintained (circled in blue in Figure $5(a))$, as will be more evident hereafter in other strain backgrounds. Nucleosome eviction is completely absent in the same conditions both in $\Delta$ atf1 cells (Figure 5(b)) [47] and in cells expressing HA-Atf1.10M (Figure 5(c)). Remodeling of the fbp1 promoter by nucleosome eviction is probably essential to allow recruitment of the large RNA polymerase II (Pol II) machinery. As shown in Figure S3, recruitment of the Pol II subunit Rpb3-HA upon glucose starvation is clear in cells expressing wildtype Atf1 or a phosphomimetic Atf1 mutant (untagged Atf1.7D, to combine it with Rpb3-HA), but not in cells expressing a hypo-phosphorylation mutant (Atf1.7M). We conclude that Atf1 phosphorylation has a key role in nucleosome eviction at the fbp 1 promoter and Pol II loading after glucose starvation, since the hypo-phosphorylation HA-Atf1.10M mutant cannot induce chromatin remodeling in this $\sim 1$ $\mathrm{kb}$ area.

\section{Constitutive phosphorylation of Atf1 accelerates chromatin remodeling of $f b p 1$ promoter to an open state}

We noticed that HA-Atf1.10D enrichment at the $f b p 1$ promoter 30 minutes after a shift to glucose-poor medium was higher compared to HA-Atf1 (Figure 4(d)), suggesting that this could affect the kinetics of fbp1 induction in speed or in magnitude. Indeed, the levels of $f b p 1$ mRNA at longer times upon glucose starvation are more sustained in cells expressing the phosphomimetic HA-Atf1.10D (Figure $6(a))$.

This observation led us to hypothesize that the phosphorylation of Atf1 previous to glucose deprivation could accelerate the opening of chromatin and this way affect the magnitude or duration of the transcriptional response. We analyzed the nucleosome eviction dynamics in cells expressing $\mathrm{HA}$ Atf1 and HA-Atf1.10D. In cells expressing wild-type HA-Atf1, the first nucleosome evicted 30 min after stress imposition is nucleosome -6 , the closest one to the Atf1-binding site, UAS1, while nucleosome -1, downstream of UAS2, is partially evicted at this time point (Figure 6(b), $30 \mathrm{~min}$, circled in red). 60 minutes after glucose depletion most nucleosomes are gone, with -3 and -5 still weakly 
positioned (Figure 6(b)). Interestingly, in glucose-rich conditions nucleosome -6 is missing in cells expressing HA-Atf1.10D strain (Figure 6(c)), even though we could not detect Atf1.10D binding to UAS1 by ChIP under basal conditions (Figure 4(d)). Only 30 minutes after glucose starvation we observed an intermediate chromatin profile, with nucleosomes -1 and -2 (surrounding UAS2) evicted, in cells expressing HA-Atf1.10D (Figure 6(c), center panel). So, these evidences suggest that constitutive phosphorylation of Atf1 promotes the early opening of chromatin surrounding fbp 1 promoter region, inducing a faster and more sustained response to glucose starvation.

Since the sequential transcription of three overlapping mlonRNA species $(-a,-b$ and $-c)$ has been reported to be essential for the opening of chromatin [33,39], we hypothesized that the faster remodeling in HA-Atf1.10D cells may be due to mlonRNAs transcription deregulation in cells expressing this phosphomimetic mutant. As reported [33], we detected induction of mlonRNAs $-b$ and $-c$ after 30 minutes of glucose starvation only in cells expressing HA-Atf1, whereas this induction was absent in cells lacking Atf1 or expressing HA-Atf1.10M, as expected. Surprisingly, induction of mlonRNAs $b$ and $c$ was absent in cells expressing the transcriptionally active HA-Atf1.10D mutant (Figure S4). We conclude that expression of the phosphomimetic mutant HA-Atf1.10D is able to promote fbp1 activation after glucose deprivation in the absence of mlonRNAs production, maybe due to the constitutive eviction of nucleosome -6 .

\section{Complete nucleosome eviction at the fbp1 promoter is necessary but not sufficient for maximal transcription activation}

As shown above, Atf1 phosphorylation is essential to initiate chromatin remodeling at the fbp 1 promoter: the HA-Atf1.10M, dead in transcription, is unable to induce nucleosome eviction, while the HA-Atf1.10D mutant, which promotes a faster chromatin remodeling than wild-type Atf1, also displays enhanced transcriptional levels. However, Atf1 phosphorylation cannot be the only factor involved, since the phosphomimetic HA-Atf1.10D mutant does not display constitutive fbp1 transcription. As explained above, the Pka1 pathway-dependent Rst2 TF is also required for fbp1 transcription (Figure 4) $[29,30]$. In glucose rich conditions, Pka1 kinase phosphorylates Rst2, keeping it excluded from the nucleus and in an inactive form. When glucose is depleted, Pka1 kinase is inactivated by Cgs1 and Rst2 is translocated to the nucleus [25]. Lack of nuclear Rst2 could be sufficient to explain the absence of constitutive fbp1 activation in cells expressing HA-Atf1.10D. We analyzed the response to 
glucose starvation in cells lacking Pka1, in which Rst2 is active also in glucose rich conditions. As previously reported [28], fbp1 expression is constitutive in strain $\Delta p k a 1$, even though further induction is accomplished in low glucose medium (Figure 7(a)). Importantly, both basal and induced fbp1 expression in cells lacking Pka1 is fully dependent on the presence of Atf1 or Sty1 [28]. The fact that strain $\Delta p k a 1$ displays constitutive fbp 1 transcription in glucose-rich conditions can only be explained if both pathways, Sty1- and Pka1-driven, are inter-connected, as previously suggested [40, 45], so that Atf1 is partially activated in this strain background.

fbp 1 is also negatively regulated by the negative TF Scr1, which has to be released from UAS2 site to allow the binding of Rst2 [35]. We tested whether the lack of Scr1 alone, or in combination with the constitutively active HA-Atf1.10D, would cause a full de-repression of $f b p 1$ in glucose-rich medium. As shown in Figure 7(b), basal levels of $f b p 1$ transcripts are 4-5 times higher in $\Delta s c r 1$ than in wild-type cells when combined with the constitutive-promoter expressed HA-Atf1, and expression of HA-Atf1.10D increases those levels two-fold. This result indicates that expression of HA-Atf1.10D in cells lacking Scr1 causes a significant increase in basal fbp1 transcription.

We tested the nucleosome architecture of the $f b p 1$ promoter in these two backgrounds, $\Delta p k a 1$ and $\Delta s c r 1$. Figure 7 (c) shows the relaxed configuration of chromatin around the fbp 1 promoter before and after glucose starvation in $\Delta$ pka 1 cells, which only display nucleosomes -3 and -5 stably positioned [compare it to the wild-type strain in Figure 5(a)]. Deletion of Scr1 is not sufficient to cause the same effect in glucose-rich medium, and only nucleosome -1, downstream of the Scr1/Rst2 binding site (UAS2) is clearly evicted prior to glucose deprivation (Figure 7 (d), circled in red). $\Delta s c r 1$ cells expressing HA-Atf1.10D display complete opening of chromatin before and after glucose deprivation (Figure $7(e)$ ), even though transcription levels are only moderately de-repressed in this background (Figure 7(b)). From these experiments we conclude that nucleosome eviction in the fbp 1 promoter, ruled by Scr1 inactivation and phosphorylated Atf1, is an essential step in transcription initiation, but the Rst2 TF, which is not activated in $\Delta s c r 1$ cells expressing HA-Atf1.10D, is essential to facilitate promoter initiation by the complex Pol II machinery and fully activate fbp 1 transcription. 


\section{DISCUSSION}

We and others have previously shown that the main role of active Sty 1 in the regulation of a wide antistress gene expression program is phosphorylating Atf1 $[10,11]$. Specifically, in response to $\mathrm{H}_{2} \mathrm{O}_{2}$, Atf1 phosphorylation promotes transcription initiation, but TF recruitment to stress promoters (1.5-3fold) is not quantitatively sufficient to explain transcription activation (2-35-fold) [11]. We showed that the effect of the Atf1 phosphorylation mutants was different depending on two subsets of stress genes, with Atf1.10M mutant being able to activate transcription in one of them (Figure 1(b)) [11]. Since Sty1 and Atf1 have been described to participate in processes other than activation of stress genes, here we have studied the role of TF phosphorylation in homologous recombination at ade6-M26 hotspot, initiation of mating and meiosis program by regulation of ste11, and fbp 1 transcription in response to glucose starvation. As will be discussed hereafter, we demonstrate that Atf1 phosphorylation by Sty1 is required in all cases. Phosphorylation at either canonical or non-canonical sites seems to be sufficient to trigger chromatin remodelling in homologous recombination and in cgs2-ste11 transcription, so that HA-Atf1.10M can initiate these processes. However, when Atf1 has to work synergistically with other TFs, such as in the case of the fbp1 promoter, not only non-canonical but also the canonical Ser/ThrP sites are required to establish all the interactions required in this complex context.

The effect of the hypo-phosphorylation mutant in cgs2-ste11-mei2 activation and ade6-M26 recombination resembles that on the gpd1 and hsp9 stress genes [11]. Atf1.10M, lacking the canonical Sty1 phosphorylation sites, is capable of activating cgs2 and its downstream ste11 and mei2 genes after nitrogen starvation (Figure 2(bcd)) and of inducing homologous recombination at ade6-M26 locus (Figure 3(c)). However, the presence of Sty1 is essential for the activity of Atf1.10M, suggesting that other non-canonical sites in Atf1 may be sufficient to promote these transcription and recombination events. The phosphomimetic Atf1.10D mutant is able to induce ste11 transcription (Figure 2(e)) and homologous recombination (Figure 3(c)) even in the absence of Sty1. This fact further supports the claim that phosphorylation of Atf1 by Sty1, either at the canonical S/TP sites or at so far unidentified residues, is the key molecular event causing activation of the TF. In all these genes/scenarios, Atf1 seems to be the only TF promoting transcription or recombination, and we propose that phosphorylation at canonical or non-canonical sites is sufficient to trigger a TF-mediated 
opening of the chromatin at those specific chromosomal locations, maybe by recruiting the Spt-AdaGcn5 acetyltransferase (SAGA) complex which has been demonstrated to regulate stress genes [48], ste11 [49] and recombination at ade6-M26 [38, 50]. Another possibility, the Sty1-dependent modification of Atf1 by a mechanism other than phosphorylation, could also explain why Sty1 is required for the activity of Atf1.10M; nevertheless, enzymatic properties other than kinase have never been attributed to this family of enzymes. Finally, hetero-dimerization with the TF Pcr1 cannot explain the differences reported here, since Pcr1 is required for homologous recombination, ste 11 and fbp 1 transcription $[51,52]$.

The situation is different at $f b p 1$. It is worth mentioning here that we have analyzed at least three cascades and downstream TFs mediating activation of fbp1: (i) the Scr1 repressor is bound to UAS2 prior to stress, and it has to be excluded from the nucleus upon glucose depletion; (ii) simultaneously, the Rst2 TF is activated upon Pka1 inactivation by low glucose, and binds to UAS2; (iii) in parallel, Atf1 has to be phosphorylated by Sty1 when glucose is depleted. In cells expressing HA-Atf1.10M, the activation of fbp1 after glucose depletion is totally suppressed (Figure 4(c), upper panel), resembling the lack of activity of this mutant at the srx 1 and ctt1 stress genes (Figure 1(b)) [11]. Also common to the effect at those genes, the HA-Atf1.10D phosphomimetic mutant cannot constitutively trigger fbp 1 expression, but has a slightly higher activity than wild-type Atf1 (Figure 4(c), lower panel). Quantitatively speaking, the recruitment of HA-Atf1 to DNA does not justify the foldtranscriptional activation, but is worth to point out that the enrichment of the TF at the fbp1 promoter region after 60 minutes of glucose starvation is abolished in cells expressing HA-Atf1.10M (Figure $4(d))$.

Contrary to the nucleosome-depleted promoters of stress genes [48], the fbp1 promoter is full of nucleosomes stably positioned prior to glucose depletion $[46,53]$ (Figure $5(a)$ ). We show here that most of them are completely evicted 60 minutes after glucose deprivation in wild-type cells, and nucleosome eviction is fully abolished in cells lacking Atf1 or expressing HA-Atf1.10M. Therefore, phosphorylation of Atf1 has an essential role in promoting the chromatin rearrangements that the Pol II machinery requires at the fbp 1 promoter. This hypothesis is further supported by the fact that nucleosome -6, the closest to the Atf1 binding site UAS1, is the first one evicted upon glucose depletion in wild-type cells (Figure 6(b), $30 \mathrm{~min}$ ), and it is not even positioned in glucose-rich medium in cells expressing HA-Atf1.10D (Figure 6(c), basal). Moreover, the absence of mlonRNAs $b$ and $c$ in 
cells expressing the phosphomimic HA-Atf1.10D could be related with the Tup co-repressors: it has been reported that cells lacking Tup11 and Tup12 do not display expression of mlonRNAs [39], and binding of the HA-Atf1.10D to UAS1 may participate in the eviction of nucleosome -6 by releasing the Tup11/Tup12 co-repressors, so that synthesis of the mlonRNAs would be dispensable. In conclusion, we propose that Atf1 phosphorylation by Sty 1 facilitates its recruitment to UAS1, from where it opens the chromatin to allow the entrance of the transcriptional machinery to trigger fbp 1 transcription.

Several experimental evidences suggest that recruitment of Atf1 to UAS1 has to be done synergistically with Rst2 binding to UAS2, causing DNA looping. First, neither wild-type Atf1 nor the phosphomimetic HA-Atf1.10D mutant are able to bind to DNA nor activate fbp1 transcription in cells lacking Rst2 (Figure 4(fg)). Second, the fbp1 gene is fully repressed in glucose-rich medium, since Rst2 is not active (Figure 4(c)). Third, expression of HA-Atf1.10D in cells lacking the repressor Scr1 only display a moderate (but not full) basal de-repression of fbp1 transcription (Figure 7(b)), even though the nucleosomes at the promoter are fully evicted in basal conditions (Figure 7(e)). Forth, the only background in which Rst2 is active in basal conditions, $\Delta$ pka1, displays (almost) full derepression in basal conditions (Figure 7(a)); we propose that in this background the Sty1-Atf1 pathway is partially engaged in the absence of stress [40], and therefore the fbp 1 promoter is constitutively free of nucleosomes (Figure 7(c)). As explained in the Introduction, it has been previously demonstrated that binding of Atf1 to UAS1 and of Rst2 to UAS2 favors the formation of a chromatin loop which spatially brings both UAS close together [30]. We propose that activated Atf1 and Rst2 associate and recognize both sites synergistically distorting the DNA between both sites through looping; the main role of Atf1 is to modify chromatin, while Rst2 probably interacts directly with Pol II or with general Pol II TFs. Additional studies will be necessary to demonstrate whether Atf1-Rst2 association occurs prior to DNA binding and DNA looping, using wild-type and mutant forms of Atf1. Regarding DNA distortion, it is worth mentioning that a recent study has demonstrated by ChIP the presence of Rst2, Atf1 and Scr1 at both the promoter and 3' region of the rsv1 gene [37]. rsv 1 and fbp 1 genes share many common regulatory features (involvement of Sty $1 /$ Atf1 and PKA pathway, activated by Atf1 and Rst2, repressed by Scr1 under glucose-rich conditions...), and therefore additional DNA looping events linking UAS1, UAS2 and the 3' gene regions could be ruling these glucose-responding genes. 
In summary, we demonstrate that Atf1 phosphorylation by Sty1 at canonical or non-canonical sites is essential for its biological roles, and the complexity of transcriptional regulation by this activated TF increases when it has to work synergistically with other TFs such as Rst2. Working alone or in combination, chromatin remodeling seems to be Atf1's main role, together with associating and promoting DNA binding of the other TFs, like Rst2 in the case of fbp 1 transcription. Whether this specific TF-TF complex is assembled prior to DNA binding or after UAS interaction will have to be analyzed. These results highlight the diverse mechanisms employed by TFs to modulate chromatin remodeling, gene expression and cell adaptation depending on the biological process regulated and on the chromatin context. 


\section{MATERIAL AND METHODS}

\section{Yeast strains, plasmids and growth conditions}

Cells were grown in rich medium (YE) or synthetic minimal medium (MM) as described previously [54]. Origins and genotypes of strains used in this study are outlined in S1 Table. We constructed the EP48 strain ( $\triangle p y p 1)$ by crossing the wild-type strain (975) with strain EP33 ( $\Delta p y p 1)$, in which the entire pyp1 open reading frame has been substituted by an antibiotic resistance cassette. To delete the atf1 genes in strains harbouring the different ade6 alleles, we transformed the strains JA123 (ade6-M26), JA126 (ade6-M375) and JA381 (ade6-M210) with linear fragments containing ORF::kanMX6, obtained by PCR amplification using ORF-specific primers and plasmid pFA6a-kanMX6 as a template, as described previously [55, 56], obtaining strains LS9 (ade6-M26), LS10 (ade6-M375) and LS13 ( $\Delta$ atf1 ade6-M210). The resulting strains were crossed out to wild-type strains to eliminate auxotrophies (except ade), yielding strains LS29, LS20 and LS50. LS9, LS10 and LS13 stains also were transformed with integrative plasmids p428' [57] and p428' mutant derivatives [11] to express HA-Atf1 and HA-Atf1 phospho-mutants under the control of the constitutive sty 1 promoter. These plasmids were inserted at the leu1-32 loci yielding the strains sets expressing HA-Atf1 or mutant derivatives LS11 (ade6-M26), LS12 (ade6-M375) and LS14 (ade6-M210). To delete sty1 in these backgrounds, we crossed strains JA123 (ade6-M26), JA126 (ade6-M375) and JA381 (ade6-M210) with EP303 (Datf1 Dsty1 HA-Atf1),

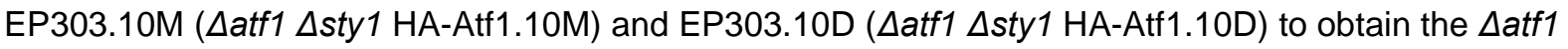
$\Delta$ sty1 strains sets expressing HA-Atf1 or mutant derivatives LS24 (ade6-M26), LS28 (ade6-M375) and LS56 (ade6-M210). We obtained the $\Delta$ atf1 $\Delta$ sty1 strains LS53 (ade6-M26), LS52 (ade6-M375) and LS59 (ade6-M210), used as negative controls, by crossing strains JA123 (ade6-M26), JA126 (ade6M375) and JA381 (ade6-M210) with EP288 ( $\Delta$ atf1 $\Delta$ sty1). 22G4 ( $\Delta$ cgs2) from the Bioneer deletion collection [58] was mated with wild-type strain (972) to generate MS110 strain ( $h$ - $\Delta c g s 2)$, which was mated with wild-type strain (975) to change the mating type, yielding LS40 ( $\left.h^{+} \Delta c g s 2\right)$ strain. By mating strain LS40 with EP193 ( $\Delta$ atf1) strain [11], we obtained the strain LS41 ( $\Delta$ atf1 $\Delta c g s 2)$. To construct

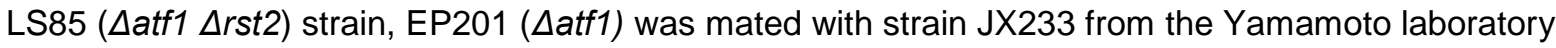
[25]. Then, the plasmids p428' and its mutant variants were also inserted at the leu1-32 loci of different strain backgrounds [LS41 ( $\Delta$ atf1 $\Delta c g s 2)$ and LS85 ( $\Delta$ atf1 $\Delta r s t 2)]$, to obtain the strain series LS46 ( $\Delta$ atf1 $\Delta c g s 2)$ and RF22 ( $\Delta$ atf1 $\Delta r s t 2)$, both of them expressing HA-Atf1, HA-Atf1.10M or HA-Atf1.10D. To 
generate AZ75 strain, we crossed the wild-type strain (972) with 25B1 strain ( $\triangle p k a 1)$ from the Bioneer deletion collection [58]. We also used the strain 25B1 ( $\Delta p k a 1)$ to delete sty1 gene with a cassette containing ura4+ gene, yielding strain AZ61 ( $\Delta p k a 1 \Delta$ sty1). We constructed strains RF94 ( $\triangle$ atf1 $\Delta p k a 1$

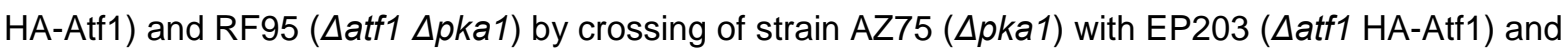

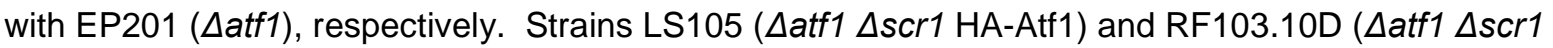

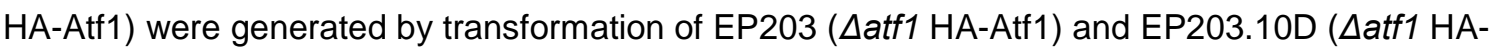
Atf1.10D) with linear fragments containing scr1::kanMX6, obtained by PCR amplification using ORFspecific primers and plasmid pFA6a-kanMX6. To tag rst2 with FLAG, we transformed EP203 series ( $\Delta$ atf1 expressing HA-Atf1 or mutant derivatives) and EP201 (Latf1) with a linear fragment containing the 3' end of rst2 fused to FLAG::kanMX6, obtained by PCR amplification using rst2 specific primers and the plasmid pFA6a-5FLAG::kanMX6, yielding the strain set LS106 (Datf1 Rst2-FLAG) expressing

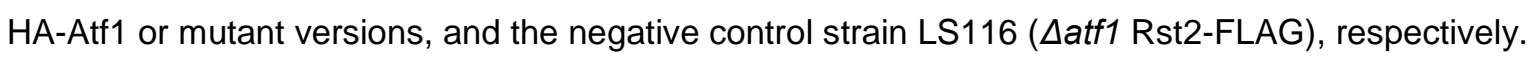

\section{Stationary phase conditions and survival assays}

Strains were grown at $30^{\circ} \mathrm{C}$ in YE medium until they reached stationary phase, at an approximate $\mathrm{OD}_{600}$ of $5-9$, depending on the strains and growth conditions. The same number of cells $\left(10^{5}-10^{1}\right)$ in 3 $\mu l$ was spotted on YE agar plates from cultures at the logarithmic phase $\left(\mathrm{OD}_{600}\right.$ of 0.5$)$ or several days after reaching the stationary phase. The spots were allowed to dry and the plates were incubated at $30^{\circ} \mathrm{C}$ for $2-4$ days.

\section{Glucose- and nitrogen- deprivation growth conditions}

To induce glucose starvation conditions, cultures of the indicated strains growing in YE with a $6 \%$ of glucose were grown until an approximate $\mathrm{OD}_{600}$ of 0.5 . The cultures were filtered and transferred to YE medium containing $0.1 \%$ of glucose and $3 \%$ of glycerol. $50 \mathrm{ml}$ samples were used for each time point. For nitrogen deprivation, cells growing in minimal medium until an approximate $\mathrm{OD}_{600}$ of 0.3 were shifted to the same medium lacking ammonium chloride by filtration. $50 \mathrm{ml}$ samples were collected at indicated times.

\section{RNA analysis by Northern Blot}


Total RNA was extracted from cultures of cells at $\mathrm{OD}_{600}$ of 0.5 by standard hot-phenol method, as described before [13]. Extracted RNA was loaded on formaldehyde agarose gels and transferred to membranes, which were hybridized with the [a-32P]-dCTP labeled fbp1, containing the complete ORF.

\section{RNA analysis by quantitative PCR}

Reverse transcription was performed with purified RNA treated with DNase I using Reverse Transcription System of Applied Biosystems (Thermo Fisher Scientific), following manufacturer's instructions. The cDNA was quantified by real-time qPCR on Light Cycler II using Light Cycler 480 SYBR Green I Master (Roche). The error bars (SD) were calculated from at least three biological replicates, unless indicated otherwise, and act1 gene was used as a control for normalization. Fold induction was calculated comparing the value of each strain and condition to that of the wild type strain during exponential growth, to which we assigned a value of 1 . Primers are listed in S2 Table.

\section{Flow cytometry}

Cells were fixed on $70 \%$ ethanol, washed with $50 \mathrm{mM}$ of sodium citrate, resuspended in $50 \mathrm{mM}$ sodium citrate and incubated with RNAse $A$ at $37^{\circ} \mathrm{C}$ overnight. About $50 \mathrm{mM}$ sodium citrate containing propidium iodide (final concentration $2.5 \mathrm{~g} / \mathrm{ml}$ ) was added to cells, which were sonicated. To determine the DNA content, the fluorescence intensities of stained cells were measured and analyzed on a FACSCalibur flow cytometer using CellQuest software.

\section{Analysis of meiotic recombinant frequencies}

Induction of mating and meiosis, as well as preparation and plating of ascospores were performed as described [54] with minor modifications. After cells mating on plates of minimal medium lacking nitrogen, the mating mixtures were resuspended and washed with $1 \%$ glucose, instead of $\mathrm{H}_{2} \mathrm{O}$. After treatment of cells mated with glucuronidase, the spore suspensions were treated with a final concentration of ethanol of $15 \%$. Spores obtained were plated immediately on YE medium in the presence and absence of adenine, and meiotic recombinant frequencies were determined by counting of total viable titer and ade6+ recombinant titer, respectively. For each cross, experimental replicate and plating condition, we counted at least 100 colonies to determine the respective titers. The 
recombinant frequency was calculated as adenine+ titer/viable titer, and data are represented as mean value and standard deviation (SD), calculated from three biological replicates.

\section{Chromatin immunoprecipitation (ChIP)}

Cells were grown in YE medium and chromatin isolation and immunoprecipitation were carried out as previously described [48], with minor modifications. Briefly, cells from $50 \mathrm{ml}$ of culture were crosslinked with $1 \%$ formaldehyde for $15 \mathrm{~min}$. After stopping cross-linking with $125 \mathrm{mM}$ glycine, pellets were lysed with a bead beater, and lysates were sonicated, yielding chromatin fragments of $\approx 400$ bp average size. Chromatin isolated was immuno-precipitated with specific antibodies [ $5 \mu \mathrm{l}$ of anti-HA antiserum (12CA5; house-made), $1 \mu \mathrm{l}$ of anti-FLAG (F7425. Sigma), or $1 \mu \mathrm{l}$ anti-phospho Ser2 CTD of Pol II (Ab5095 Abcam)] overnight at $4^{\circ} \mathrm{C}$ rotating. Beads were washed, DNA was eluted and formaldehyde cross-linking was reversed. After protein digestion and chromatin extraction, DNA was amplified by quantitative real-time qPCR using Light Cycler 480 SYBR Green IMaster (Roche). The error bars (SD) were calculated from at least three biological triplicates, unless indicated otherwise and act1 gene was used as a negative control. The primers used are listed in S2 Table.

\section{Nucleosome-scanning analysis}

Mononucleosomes were obtained as described previously [48] and the resulting DNA was analyzed by RT-qPCR as described before. Briefly, cells growing in $250 \mathrm{ml}$ of YE medium to an $\mathrm{OD}_{600}$ of 0.5 were cross-linked with $0.5 \%$ formaldehyde for $20 \mathrm{~min}$ at $25^{\circ} \mathrm{C}$. Spheroplasts were obtained by digestion with zymolyase 20T (Amsbio) and treated with increasing concentrations of micrococcal nuclease (MNase; Sigma). After DNA purification and electrophoretic separation, samples displaying $80-90 \%$ mononucleosomal DNA without subnucleosomal fragments (faster migration than mononucleosomes in the electrophoresis) were further analysed by RT-qPCR with a set of overlapping primer pairs (see S2 Table). For each primer pair, numbers in Y-axis correspond to the relative value to the input, which was obtained using as a template DNA from cells not treated with MNase, and received a value of 1 . 


\section{ACKNOWLEDGEMENTS}

We are thankful to Dr. Masayuki Yamamoto for providing strain JX233. This work is supported by the Ministerio de Ciencia, Innovación y Universidades (Spain), PLAN E and FEDER (PGC2018-093920-

B-I00 to E.H.). The Oxidative Stress and Cell Cycle group is also supported by Generalitat de Catalunya (Spain) (2017-SGR-539) and by Unidad de Excelencia María de Maeztu, funded by the AEI (CEX2018-000792-M) (Spain). R.F. is recipient of an FPI predoctoral fellowship by the Ministerio de Ciencia, Innovación y Universidades (Spain). E.H. is recipient of an ICREA Academia Award (Generalitat de Catalunya, Spain).

\section{AUTHOR CONTRIBUTIONS}

All authors designed and analyzed the data. L.S.-M. and R.F. conducted the experiments. E.H. and J.A. supervised the work, and L.S.-M. and E.H. wrote the manuscript.

\section{APPENDIX A: SUPPLEMENTARY DATA}

Supplementary data to this article can be found online

Declarations of interest: none 


\section{REFERENCES}

[1] Ho YH, Gasch AP. Exploiting the yeast stress-activated signaling network to inform on stress biology and disease signaling. Curr Genet. 2015;61:503-11.

[2] Weake VM, Workman JL. Inducible gene expression: diverse regulatory mechanisms. Nat Rev Genet. 2010;11:426-37.

[3] Shiozaki K, Russell P. Cell-cycle control linked to extracellular environment by MAP kinase pathway in fission yeast. Nature. 1995;378:739-43.

[4] Samejima I, Mackie S, Fantes PA. Multiple modes of activation of the stress-responsive MAP kinase pathway in fission yeast. EMBO J. 1997;16:6162-70.

[5] Chen D, Toone WM, Mata J, Lyne R, Burns G, Kivinen K, et al. Global transcriptional responses of fission yeast to environmental stress. MolBiolCell. 2003;14:214-29.

[6] Chen D, Wilkinson CR, Watt S, Penkett CJ, Toone WM, Jones N, et al. Multiple pathways differentially regulate global oxidative stress responses in fission yeast. Mol Biol Cell. 2008;19:308-17.

[7] Millar JB, Buck V, Wilkinson MG. Pyp1 and Pyp2 PTPases dephosphorylate an osmosensing MAP kinase controlling cell size at division in fission yeast. Genes Dev. 1995;9:2117-30.

[8] Shiozaki K, Russell P. Counteractive roles of protein phosphatase 2C (PP2C) and a MAP kinase kinase homolog in the osmoregulation of fission yeast. EMBO J. 1995;14:492-502.

[9] Shiozaki K, Russell P. Conjugation, meiosis, and the osmotic stress response are regulated by Spc1 kinase through Atf1 transcription factor in fission yeast. Genes Dev. 1996;10:2276-88.

[10] Wilkinson MG, Samuels M, Takeda T, Toone WM, Shieh JC, Toda T, et al. The Atf1 transcription factor is a target for the Sty1 stress-activated MAP kinase pathway in fission yeast. Genes Dev. 1996;10:2289-301.

[11] Salat-Canela C, Paulo E, Sanchez-Mir L, Carmona M, Ayte J, Oliva B, et al. Deciphering the role of the signal- and Sty 1 kinase-dependent phosphorylation of the stress-responsive transcription factor Atf1 on gene activation. J Biol Chem. 2017;292:13635-44.

[12] Sanso M, Gogol M, Ayte J, Seidel C, Hidalgo E. Transcription factors Pcr1 and Atf1 have distinct roles in stress- and Sty1-dependent gene regulation. Eukaryot Cell. 2008;7:826-35.

[13] Castillo EA, Vivancos AP, Jones N, Ayte J, Hidalgo E. Schizosaccharomyces pombe cells lacking the Ran-binding protein Hba1 show a multidrug resistance phenotype due to constitutive nuclear accumulation of Pap1. J Biol Chem. 2003;278:40565-72.

[14] Lawrence CL, Maekawa H, Worthington JL, Reiter W, Wilkinson CR, Jones N. Regulation of Schizosaccharomyces pombe Atf1 protein levels by Sty1-mediated phosphorylation and heterodimerization with Pcr1. J Biol Chem. 2007;282:5160-70.

[15] Sanchez-Mir L, Salat-Canela C, Paulo E, Carmona M, Ayte J, Oliva B, et al. Phospho-mimicking Atf1 mutants bypass the transcription activating function of the MAP kinase Sty1 of fission yeast. Curr Genet. 2018;64:97-102.

[16] Wahls WP, Smith GR. A heteromeric protein that binds to a meiotic homologous recombination hot spot: correlation of binding and hot spot activity. Genes Dev. 1994;8:1693-702.

[17] Steiner WW, Smith GR. Natural meiotic recombination hot spots in the Schizosaccharomyces pombe genome successfully predicted from the simple sequence motif M26. Mol Cell Biol.

2005;25:9054-62. 
[18] Wahls WP, Davidson MK. Discrete DNA sites regulate global distribution of meiotic recombination. Trends Genet. 2010;26:202-8.

[19] Steiner WW, Schreckhise RW, Smith GR. Meiotic DNA breaks at the S. pombe recombination hot spot M26. Mol Cell. 2002;9:847-55.

[20] Gao J, Davidson MK, Wahls WP. Phosphorylation-independent regulation of Atf1-promoted meiotic recombination by stress-activated, p38 kinase Spc1 of fission yeast. PLoS One. 2009;4:e5533.

[21] Maeda T, Mochizuki N, Yamamoto M. Adenylyl cyclase is dispensable for vegetative cell growth in the fission yeast Schizosaccharomyces pombe. Proc Natl Acad Sci U S A. 1990;87:7814-8.

[22] Sugimoto A, lino Y, Maeda T, Watanabe $Y$, Yamamoto M. Schizosaccharomyces pombe ste11+ encodes a transcription factor with an HMG motif that is a critical regulator of sexual development. Genes Dev. 1991;5:1990-9.

[23] Mochizuki N, Yamamoto M. Reduction in the intracellular cAMP level triggers initiation of sexual development in fission yeast. Mol Gen Genet. 1992;233:17-24.

[24] Kjaerulff S, Andersen NR, Borup MT, Nielsen O. Cdk phosphorylation of the Ste11 transcription factor constrains differentiation-specific transcription to G1. Genes Dev. 2007;21:347-59.

[25] Higuchi T, Watanabe Y, Yamamoto M. Protein kinase A regulates sexual development and gluconeogenesis through phosphorylation of the $\mathrm{Zn}$ finger transcriptional activator Rst2p in fission yeast. MolCell Biol. 2002;22:1-11.

[26] Eshaghi M, Lee JH, Zhu L, Poon SY, Li J, Cho KH, et al. Genomic binding profiling of the fission yeast stress-activated MAPK Sty1 and the bZIP transcriptional activator Atf1 in response to H2O2. PLoS One. 2010;5:e11620.

[27] Davidson MK, Shandilya HK, Hirota K, Ohta K, Wahls WP. Atf1-Pcr1-M26 complex links stressactivated MAPK and cAMP-dependent protein kinase pathways via chromatin remodeling of cgs $2+. \mathrm{J}$ Biol Chem. 2004;279:50857-63.

[28] Hoffman CS, Winston F. Glucose repression of transcription of the Schizosaccharomyces pombe fbp1 gene occurs by a cAMP signaling pathway. Genes Dev. 1991;5:561-71.

[29] Hirota K, Hasemi T, Yamada T, Mizuno KI, Hoffman CS, Shibata T, et al. Fission yeast global repressors regulate the specificity of chromatin alteration in response to distinct environmental stresses. Nucleic Acids Res. 2004;32:855-62.

[30] Asada R, Umeda M, Adachi A, Senmatsu S, Abe T, Iwasaki H, et al. Recruitment and delivery of the fission yeast Rst2 transcription factor via a local genome structure counteracts repression by Tup1-family corepressors. Nucleic Acids Res. 2017;45:9361-71.

[31] Neely LA, Hoffman CS. Protein kinase A and mitogen-activated protein kinase pathways antagonistically regulate fission yeast fbp 1 transcription by employing different modes of action at two upstream activation sites. MolCell Biol. 2000;20:6426-34.

[32] Hirota K, Mizuno K, Shibata T, Ohta K. Distinct chromatin modulators regulate the formation of accessible and repressive chromatin at the fission yeast recombination hotspot ade6-M26. Mol Biol Cell. 2008;19:1162-73.

[33] Senmatsu S, Asada R, Abe T, Hoffman CS, Ohta K, Hirota K. IncRNA transcriptional initiation induces chromatin remodeling within a limited range in the fission yeast fbp1 promoter. Scientific reports. 2019;9:299.

[34] Takemata N, Oda A, Yamada T, Galipon J, Miyoshi T, Suzuki Y, et al. Local potentiation of stress-responsive genes by upstream noncoding transcription. Nucleic Acids Res. 2016;44:5174-89. 
[35] Hirota K, Hoffman CS, Ohta K. Reciprocal nuclear shuttling of two antagonizing Zn finger proteins modulates Tup family corepressor function to repress chromatin remodeling. Eukaryot Cell. 2006;5:1980-9.

[36] Saitoh S, Mori A, Uehara L, Masuda F, Soejima S, Yanagida M. Mechanisms of expression and translocation of major fission yeast glucose transporters regulated by CaMKK/phosphatases, nuclear shuttling, and TOR. Mol Biol Cell. 2015;26:373-86.

[37] Kim EJ, Cho YJ, Chung WH, Roe JH. The role of Rsv1 in the transcriptional regulation of genes involved in sugar metabolism for long-term survival. FEBS J. 2020;287:878-96.

[38] Yamada T, Mizuno K, Hirota K, Kon N, Wahls WP, Hartsuiker E, et al. Roles of histone acetylation and chromatin remodeling factor in a meiotic recombination hotspot. Embo $\mathrm{J}$. 2004;23:1792-803.

[39] Hirota K, Miyoshi T, Kugou K, Hoffman CS, Shibata T, Ohta K. Stepwise chromatin remodelling by a cascade of transcription initiation of non-coding RNAs. Nature. 2008;456:130-4.

[40] Zuin A, Carmona M, Morales-Ivorra I, Gabrielli N, Vivancos AP, Ayte J, et al. Lifespan extension by calorie restriction relies on the Sty1 MAP kinase stress pathway. Embo J. 2010;29:981-91.

[41] Zuin A, Castellano-Esteve D, Ayte J, Hidalgo E. Living on the edge: stress and activation of stress responses promote lifespan extension. Aging (Albany NY). 2010;2:231-7.

[42] Kanoh J, Watanabe Y, Ohsugi M, lino Y, Yamamoto M. Schizosaccharomyces pombe gad7+ encodes a phosphoprotein with a bZIP domain, which is required for proper G1 arrest and gene expression under nitrogen starvation. Genes Cells. 1996;1:391-408.

[43] Kettenbach AN, Deng L, Wu Y, Baldissard S, Adamo ME, Gerber SA, et al. Quantitative phosphoproteomics reveals pathways for coordination of cell growth and division by the conserved fission yeast kinase pom1. Mol Cell Proteomics. 2015;14:1275-87.

[44] Koch A, Krug K, Pengelley S, Macek B, Hauf S. Mitotic substrates of the kinase aurora with roles in chromatin regulation identified through quantitative phosphoproteomics of fission yeast. Sci Signal. 2011;4:rs6.

[45] Kon N, Schroeder SC, Krawchuk MD, Wahls WP. Regulation of the Mts1-Mts2-dependent ade6M26 meiotic recombination hot spot and developmental decisions by the Spc1 mitogen-activated protein kinase of fission yeast. MolCell Biol. 1998;18:7575-83.

[46] Gonzalez S, Garcia A, Vazquez E, Serrano R, Sanchez M, Quintales L, et al. Nucleosomal signatures impose nucleosome positioning in coding and noncoding sequences in the genome. Genome Res. 2016;26:1532-43.

[47] Asada R, Takemata N, Hoffman CS, Ohta K, Hirota K. Antagonistic controls of chromatin and mRNA start site selection by Tup family corepressors and the CCAAT-binding factor. Mol Cell Biol. 2015;35:847-55.

[48] Sanso M, Vargas-Perez I, Quintales L, Antequera F, Ayte J, Hidalgo E. Gcn5 facilitates Pol II progression, rather than recruitment to nucleosome-depleted stress promoters, in

Schizosaccharomyces pombe. Nucleic Acids Res. 2011;39:6369-79.

[49] Helmlinger D, Marguerat S, Villen J, Gygi SP, Bahler J, Winston F. The S. pombe SAGA complex controls the switch from proliferation to sexual differentiation through the opposing roles of its subunits Gcn5 and Spt8. Genes Dev. 2008;22:3184-95.

[50] Mukiza TO, Protacio RU, Davidson MK, Steiner WW, Wahls WP. Diverse DNA Sequence Motifs Activate Meiotic Recombination Hotspots Through a Common Chromatin Remodeling Pathway.

Genetics. 2019;213:789-803. 
[51] Watanabe Y, Yamamoto M. Schizosaccharomyces pombe pcr1+ encodes a CREB/ATF protein involved in regulation of gene expression for sexual development. Mol Cell Biol. 1996;16:704-11.

[52] Kon N, Krawchuk MD, Warren BG, Smith GR, Wahls WP. Transcription factor Mts1/Mts2 (Atf1/Pcr1, Gad7/Pcr1) activates the M26 meiotic recombination hotspot in Schizosaccharomyces pombe. Proc Natl Acad Sci U S A. 1997;94:13765-70.

[53] Lantermann AB, Straub T, Stralfors A, Yuan GC, Ekwall K, Korber P. Schizosaccharomyces pombe genome-wide nucleosome mapping reveals positioning mechanisms distinct from those of Saccharomyces cerevisiae. Nat Struct Mol Biol. 2010;17:251-7.

[54] Alfa C, Fantes P, Hyams J, McLeod M, Warbrick E. Experiments with Fission Yeast: A Laboratory Course Manual. Cold Spring Harbor, N.Y.: Cold Spring Harbor Laboratory; 1993.

[55] Castillo EA, Ayte J, Chiva C, Moldon A, Carrascal M, Abian J, et al. Diethylmaleate activates the transcription factor Pap1 by covalent modification of critical cysteine residues. Mol Microbiol. 2002;45:243-54.

[56] Vivancos AP, Castillo EA, Biteau B, Nicot C, Ayte J, Toledano MB, et al. A cysteine-sulfinic acid in peroxiredoxin regulates $\mathrm{H} 2 \mathrm{O} 2$-sensing by the antioxidant Pap1 pathway. Proc Natl Acad Sci U S A. 2005; 102:8875-80.

[57] Garcia P, Paulo E, Gao J, Wahls WP, Ayte J, Lowy E, et al. Binding of the transcription factor Atf 1 to promoters serves as a barrier to phase nucleosome arrays and avoid cryptic transcription. Nucleic Acids Res. 2014;42:10351-9.

[58] Roguev A, Wiren M, Weissman JS, Krogan NJ. High-throughput genetic interaction mapping in the fission yeast Schizosaccharomyces pombe. Nat Methods. 2007;4:861-6. 


\section{FIGURES}

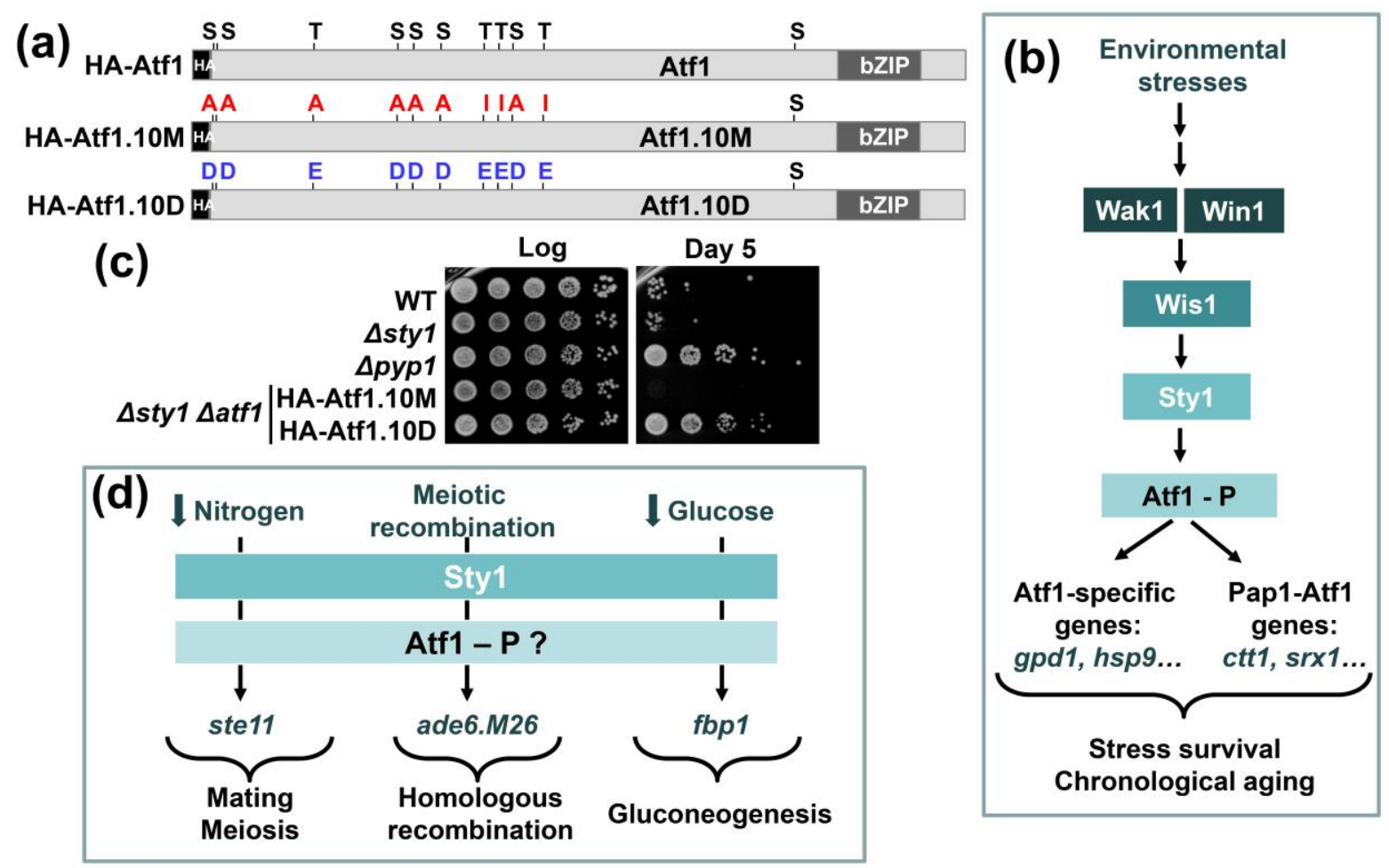

Figure 1. Atf1 phosphorylation by MAPK Sty1 is essential for its activation as a transcription factor. (a) Scheme depicting the putative MAPK phosphorylation sites, and their modifications to imitate nonphosphorylated status (10M) and constitutively phosphorylated (10D) respectively. (b) MAP kinase module of the Sty1/Atf1 pathway, including the redundant triple MAP kinases Wak1 and Win1, the MAPK kinase Wis1, MAP kinase Sty1 and the main TF, Atf1. Groups of differently regulated genes, one of them further regulated by the TF Pap1, and their biological outcome are also shown. (c) Serial dilutions of the strains 972 (WT), AV18 ( $\Delta$ sty 1$)$, EP48 ( $\Delta$ pyp1), EP303.10M ( $\Delta$ atf1 $\Delta$ sty $1+$ HAAtf1.10M) and EP303.10D ( $\Delta$ atf1 $\Delta$ sty1 + HA-Atf1.10D), growing in YE liquid medium at the logarithmic phase or 5 days after reaching stationary phase, were spotted in YE plates. (d) Scheme proposing the participation of Sty1-mediated phosphorylation of Atf1 on different biological processes, including mating and meiosis, recombination and gluconeogenesis. 


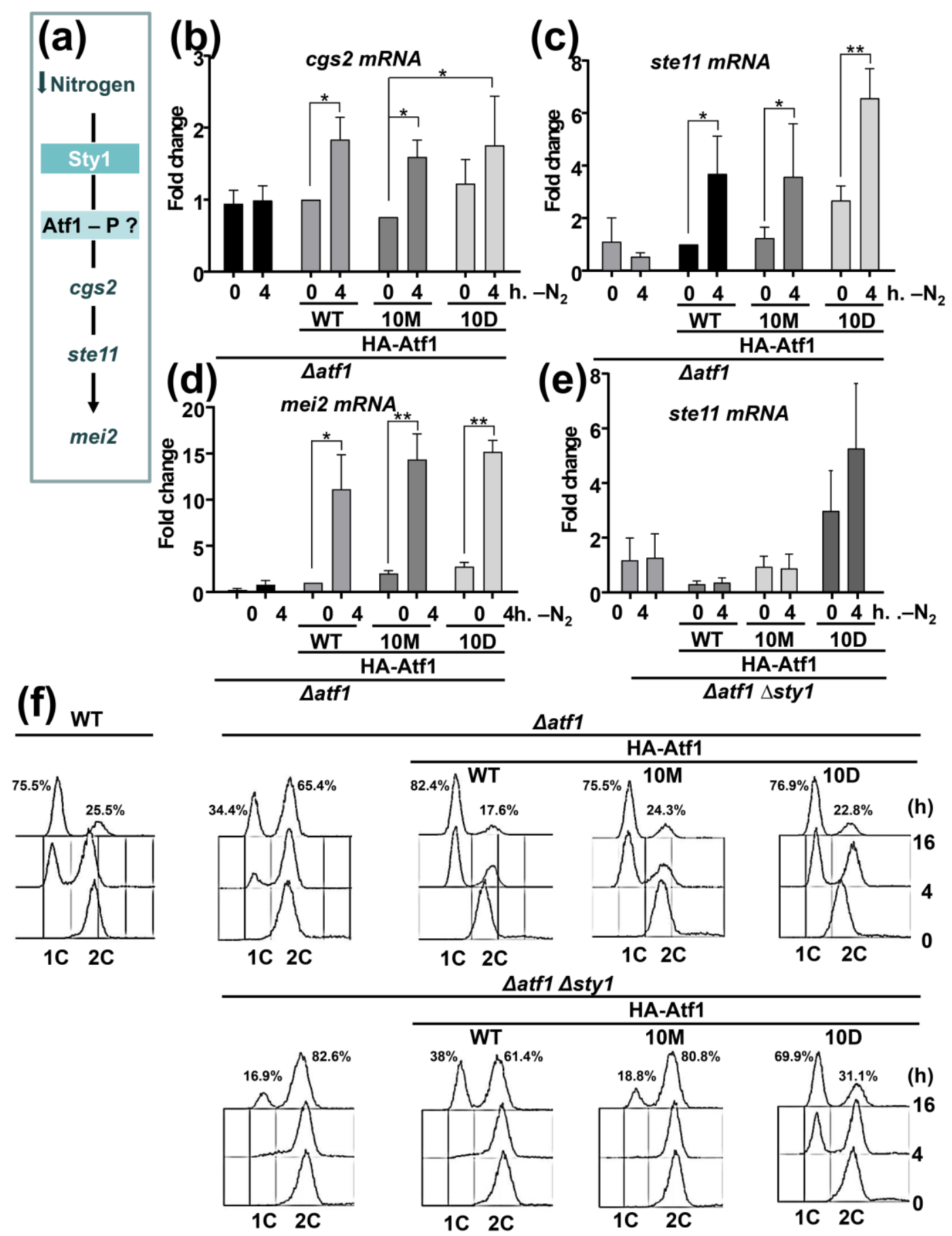

Figure 2. Cells expressing HA-Atf1.10M are still able to activate ste11 after nitrogen starvation, but in a Sty1-dependent manner. (a) Scheme of nitrogen depletion-, Sty1- and Atf1-dependent regulation of cgs2, ste 11 and mei2 transcription. (b, c and d) Expression of cgs2, ste11 and mei2 was analyzed by RT-qPCR in the strains EP201 ( $\triangle$ atf1 + empty plasmid), EP203 ( $\triangle$ atf1 + WT HA-Atf1), EP203.10M $(\triangle$ atf1 + HA-Atf1.10M) and EP203.10D ( $\triangle$ atf1 + HA-Atf1.10D). Amplification with act1 primers was used as a control for normalization. Each column represents the mean value and SD, calculated from three biological replicates. ${ }^{*}, p<0.05 ;{ }^{* *}, p<0.01$ (Student's $t$ test). (e) Expression of ste 11 was analyzed by RT-qPCR as in B, C and D, from strains EP288 ( $\Delta$ sty $1 \Delta$ atf1 + empty plasmid), EP303 ( $\Delta$ sty1 $\Delta$ atf1 + WT HA-Atf1), EP303.10M ( $\Delta$ sty1 $\Delta$ atf1 + HA-Atf1.10M), EP303.10D ( $\Delta$ sty1 $\Delta$ atf1 + HA-Atf1.10D). (f) Accumulation in G1 phase was studied by analysis of the DNA content by flow cytometry from minimal medium cultures of 972 (WT) and strains as in (b, c), before and after 4 and $16 \mathrm{~h}$ of a shift to nitrogen depleted medium assays. Histograms represent the percentage of cells with DNA content $1 \mathrm{C}$ or $2 \mathrm{C}$. 


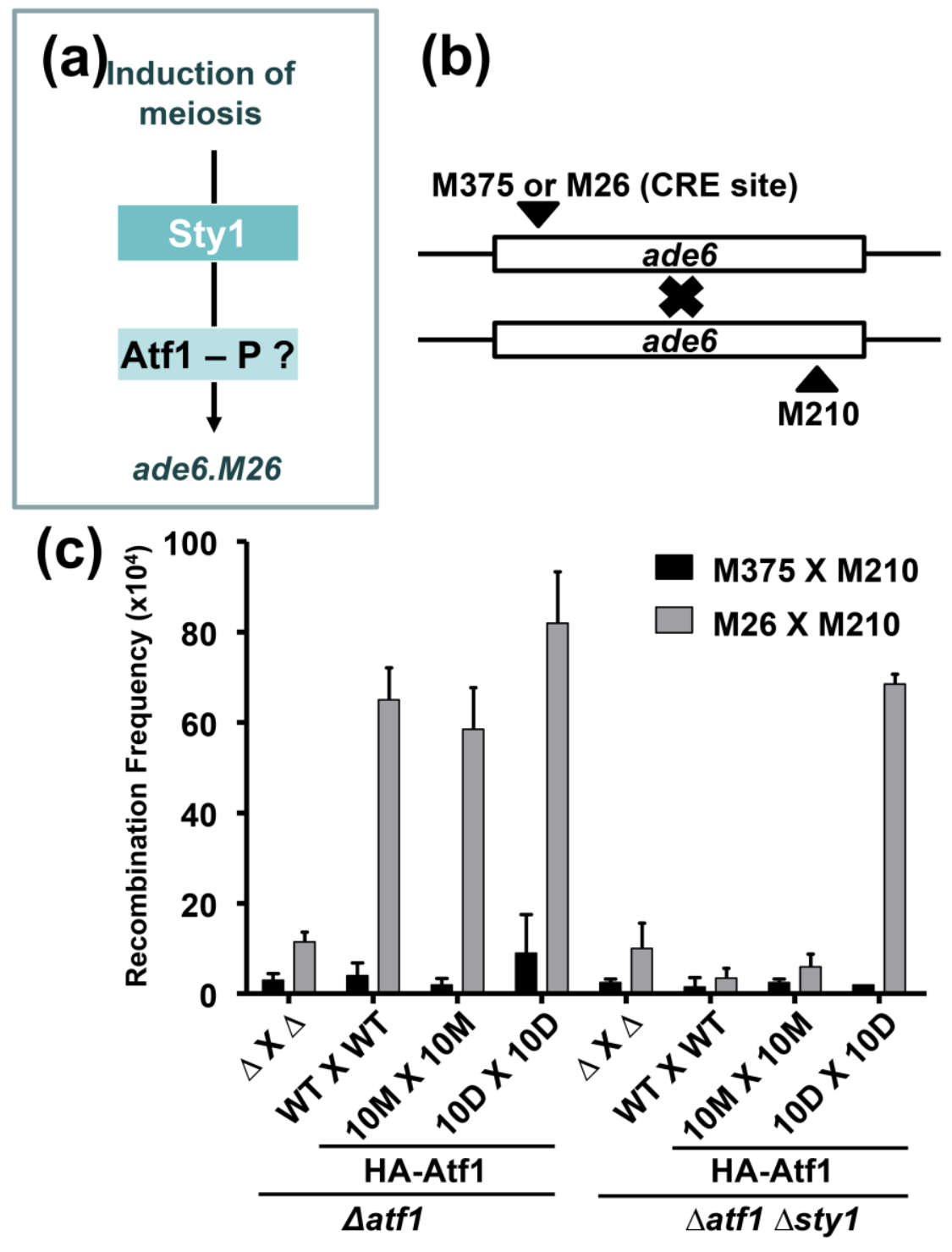

Figure 3. HA-Atf1.10M is able to induce recombination at the ade6-M26 hotspot, but only when Sty1 is present. ( $a$ and $b)$ Atf1 and the Sty1 MAPK pathway are key factors in meiotic recombination. The ade6-M26 allele contains a DNA binding site for the Atf1-Pcr1 heterodimer, whereas the ade6-M375 and ade6-M210 alleles do not. (c) In order to analyze the effect of Atf1 phosphorylation in recombination, we used the following strains : LS19 ( $h+\Delta$ atf1 ade6-M26), LS50 ( $h$ - $\Delta$ atf1 ade6-M210), LS11 $\left(h^{+} \Delta\right.$ atf1 ade6-M26 + HA-Atf1), LS11.10M ( $h+\Delta$ atf1 ade6-M26 + HA-Atf1.10M), LS11.10D $\left(h^{+}\right.$ $\Delta$ atf1 ade6-M26 + HA-Atf1.10D), LS12 ( $h+\Delta$ atf1 ade6-M375 + HA-Atf1), LS12.10M ( $h^{+} \Delta$ atf1 ade6M375 + HA-Atf1.10M), LS12.10D ( $h^{+} \Delta$ atf1 ade6-M375 + HA-Atf1.10D), LS14 $(h-\Delta$ atf1 ade6-M210 + HA-Atf1), LS14.10M ( $h$ - $\Delta$ atf1 ade6-M210 + HA-Atf1.10M), LS14.10D ( $h$ - $\Delta$ atf1 ade6-M210 + HAAtf1.10D), LS53 ( $h^{+} \Delta$ sty1 $\Delta$ atf1 ade6-M26 + empty plasmid), LS59 $(h-\Delta$ sty1 $\Delta$ atf1 ade6-M210 + empty plasmid), LS24 ( $h^{+} \Delta$ sty1 $\Delta$ atf1 ade6-M26 + HA-Atf1), LS24.10M $\left(h^{+} \Delta\right.$ sty $1 \Delta$ atf1 ade6-M26 + HA-Atf1.10M), LS24.10D ( $h^{+} \Delta$ sty1 $\Delta$ atf1 ade6-M26 + HA-Atf1.10D), LS28 ( $h^{+} \Delta$ sty1 $\Delta$ atf1 ade6-M375 + HA-Atf1), LS28.10M ( $h^{+} \Delta$ sty1 $\Delta$ atf1 ade6-M375 + HA-Atf1.10M), LS28.10D ( $h+\Delta$ sty1 $\Delta$ atf1 ade6M375 + HA-Atf1.10D), LS56 ( $h$ - $\Delta$ sty1 $\Delta$ atf1 ade6-M210 + HA-Atf1), LS56.10M ( $h$ - $\Delta$ sty1 $\Delta$ atf1 ade6M210 + HA-Atf1.10M), LS56.10D ( $h$ - $\Delta$ sty1 $\Delta$ atf1 ade6-M210 + HA-Atf1.10D). Mating between cells containing M375 and M210 sites serves as a control of basal recombination, while crosses involving strains with a mutation in M26 serve as a site-dependent recombination reporter. Data are mean +/SD from three independent experiments. 


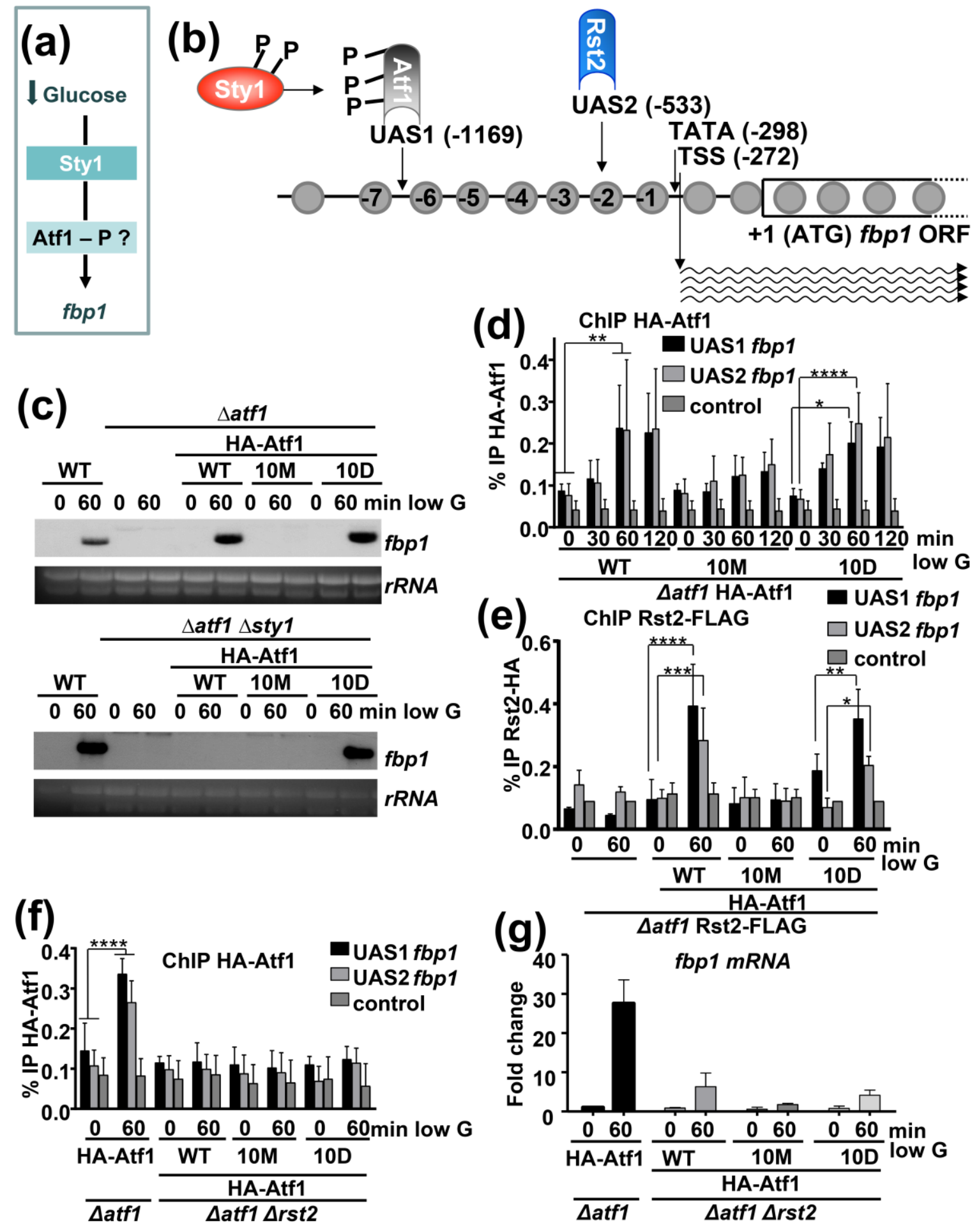

Figure 4. Atf1 phosphorylation is essential for fbp 1 induction. (a) Scheme of glucose depletion-, Sty1- and Atf1-dependent regulation of fbp 1 transcription. (b) Scheme depicting the fbp 1 promoter and open reading frame regions, including the relative localization of the nucleosomes, the binding sites of the TFs Atf1 (UAS1) and Rst2 (UAS2), the transcription start site (TSS) and the TATA box. Numbers refer to the translation start site. (c) Total RNA from cultures of the strains 972 (wild type),

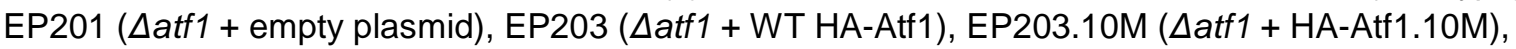

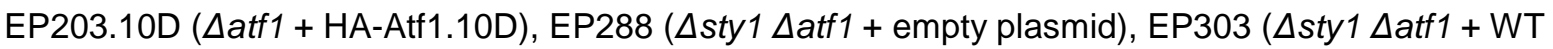
HA-Atf1), EP303.10M ( $\Delta$ sty1 $\Delta$ atf1 + HA-Atf1.10M), EP303.10D ( $\Delta$ sty1 $\Delta$ atf1 + HA-Atf1.10D) was obtained, either in exponential growth in YE with $6 \%$ glucose or after 60 minutes of growth in YE with 
$0.08 \%$ glucose, was analyzed by Northern blot with labeled fbp 1 probe . rRNA was used as loading control. (d) ChIP experiment using anti-HA antibody to localize HA-Atf1 mutants binding to UAS1 and UAS2 sites in the fbp1 promoter after glucose depletion. Samples from strains EP203 ( $\triangle$ atf1 + WT

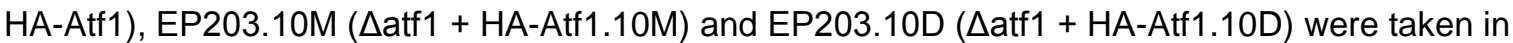
exponential growth, or 30,60 or $120 \mathrm{~min}$ after switching the cultures to low-glucose medium. Primers covering the UAS1 and UAS2 were used. Data are presented as mean +/- SD; ${ }^{*}, p<0.05 ;{ }^{* *}, p<0.01$; ${ }^{* * *}, p<0.001 ; * * * *, p<0.0001$ (Student's t test). (e) ChIP experiment using anti-FLAG antibody to localize Rst2-FLAG. Samples were obtained from cultures of the strains LS116 ( $\triangle$ atf1 rst2-FLAG with

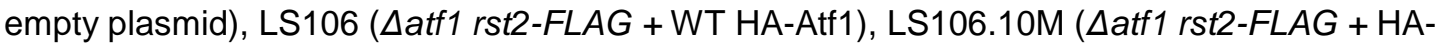
Atf1.10M) and LS106.10D ( $\triangle$ atf1 rst2-FLAG + HA-atf1.10D) and LS116 ( $\triangle$ atf1 rst2-FLAG with empty plasmid), either from exponential cultures of after 60 minutes of glucose deprivation. Data are presented as mean $+/-\mathrm{SD} ;{ }^{*}, p<0.05 ;{ }^{* *}, p<0.01 ;{ }^{* * *}, p<0.001 ;{ }^{* * * *}, p<0.0001$ (Student's t test). (f) ChIP experiment as performed in D, from strains EP203 ( $\Delta$ atf1 + HA-Atf1), RF22 ( $\Delta$ rst2 $\Delta$ atf1 + WT

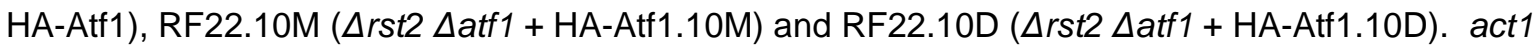
gene was used as negative control. Data are presented as mean $+/-\mathrm{SD} ;{ }^{* * *}, p<0.0001$ (Student's $t$ test). (g) fbp1 expression from the strains and conditions as in f was analyzed by RT-qPCR. 


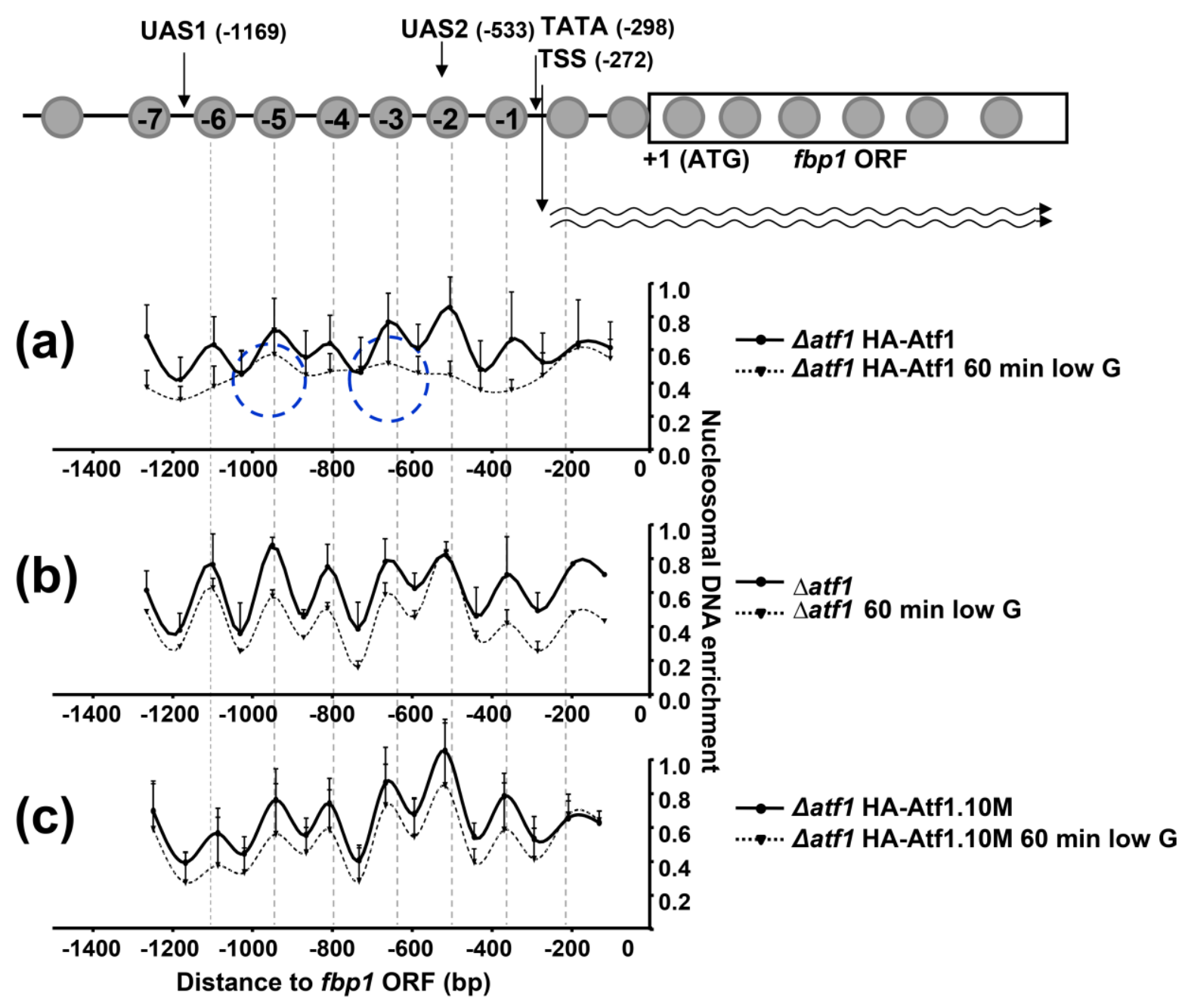

Figure 5. Nucleosome eviction from the $f b p 1$ promoter after glucose starvation depends on Atf1 phosphorylation. (a-c) Mononucleosomes of strains EP203 ( $\triangle$ atf1 + HA-Atf1) (a), EP201 ( $\triangle$ atf1 + empty plasmid) (b) and EP203.10M ( $\triangle$ atf1 + HA-Atf1.10M) (c) were isolated from logarithmic cultures or 60 minutes after switching to low glucose medium. RT-qPCR was performed using 16 pairs of primers covering $1.2 \mathrm{~kb}$ of the fbp1 promoter, including TSS, UAS1 and UAS2 as seen in the upper scheme. The values of nucleosome occupancy for each RT-qPCR reaction (Y-axis) is plotted against the gene position of the PCR-amplified fragment for each primers pair, relative to the first nucleotide of the ORF, with a value of 0 (X-axis). Mean of three biological replicates is shown, with error bars reflecting SD. Vertical dotted lines correlate to nucleosome peaks based on a genome-wide study [46]. Blue circles indicate nucleosomes -3 and -5 , which are not evicted as efficiently as the other nucleosomes. 
(a)
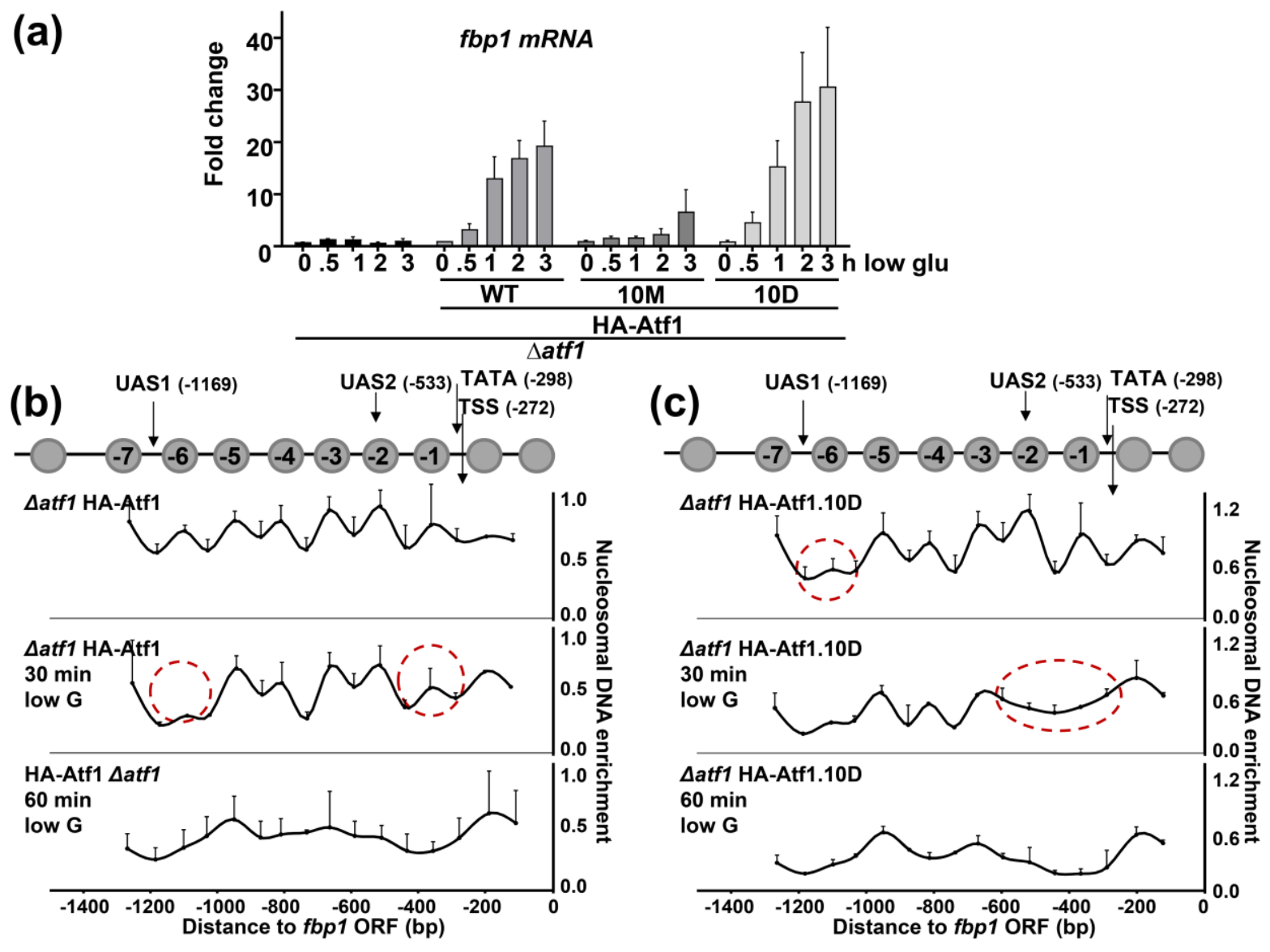

Figure 6. Constitutive phosphorylation of Atf1 accelerates the chromatin remodeling at $f b p 1$ to an open state. (a) RNA from cultures of the following strains was obtained during exponential growth and $30,60,120$ and 180 minutes after switching the culture to $0.08 \%$ glucose medium: EP201 ( $\triangle$ atf1

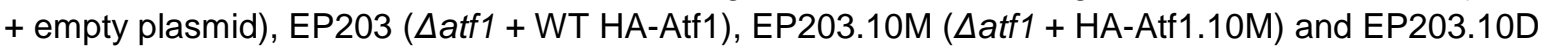
( $\triangle$ atf1 + HA-Atf1.10D). RNA was extracted and analyzed with fbp1 primers, and normalized with act1. Fold induction levels were calculated by assigning a value of 1 to fbp 1 levels in a wild type strain during exponential growth. ( $b$ and $c$ ) Time course nucleosome scanning experiments. Nucleosomes

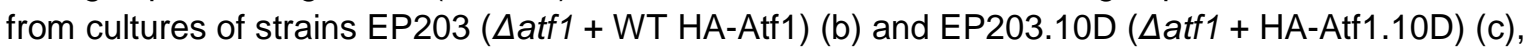
grown at exponential phase or 30 and 60 minutes after depletion of glucose, were isolated and analyzed as in Figure 5. Dotted red circles indicate nucleosomes -6 and -1 , the closer nucleosomes to UAS1 and UAS2 respectively, and the first ones evicted after nucleosome remodeling at $30 \mathrm{~min}$ in wild-cells (b), or earlier in HA-Atf1.10D expressing cells (c). 

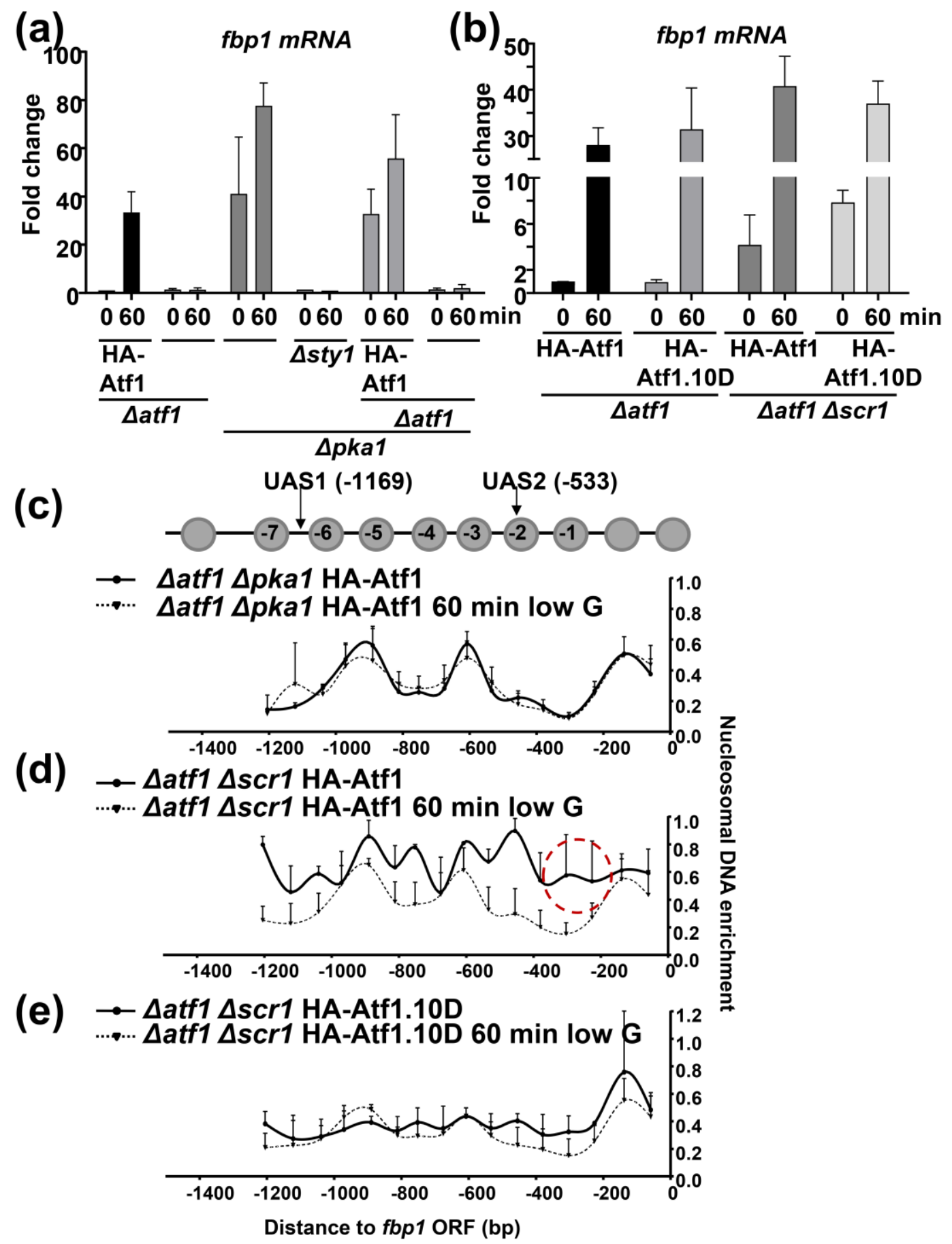

Figure 7. Role of Pka1 kinase and Scr1 repressor in fbp1 promoter chromatin remodeling. ( $a$ and $b$ ) Expression of fbp1 was analyzed by RT-qPCR in strains EP203 ( $\triangle$ atf1 + HA-Atf1), EP201 ( $\triangle$ atf1 + empty plasmid), AZ75 ( $\Delta p k a 1)$, AZ61 ( $\Delta$ pka1 $\Delta$ sty1), RF94 ( $\Delta p k a 1 \Delta$ atf1 + HA-Atf1), RF95 ( $\Delta$ pka1 $\Delta$ atf1 + empty plasmid), EP203.10D ( $\Delta$ atf1 + HA-Atf1.10D), LS105 ( $\Delta$ atf1 $\Delta s c r 1+$ HA-Atf1) and RF103.10D ( $\Delta$ atf1 $\Delta s c r 1+$ HA-Atf1.10D). Samples were obtained from exponential $6 \%$ glucose YE cultures or 60 minutes after switching them to $0.08 \%$ glucose YE medium. We show the mean and $\mathrm{SD}$ from three biological replicates. Fold induction was calculated compared to fbp 1 levels in a EP203 strain during exponential growth. (c-e) Nucleosome positioning experiments in cells lacking

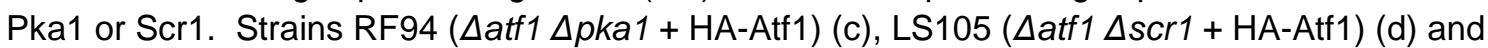
RF103.10D ( $\Delta$ atf1 $\Delta s c r 1+$ HA-Atf1.10D) (e) were grown and nucleosome positioning determined as described in Figure 5 . Dotted red circle in (D) indicates nucleosome -1, constitutively evicted in cells lacking the repressor Scr1. 

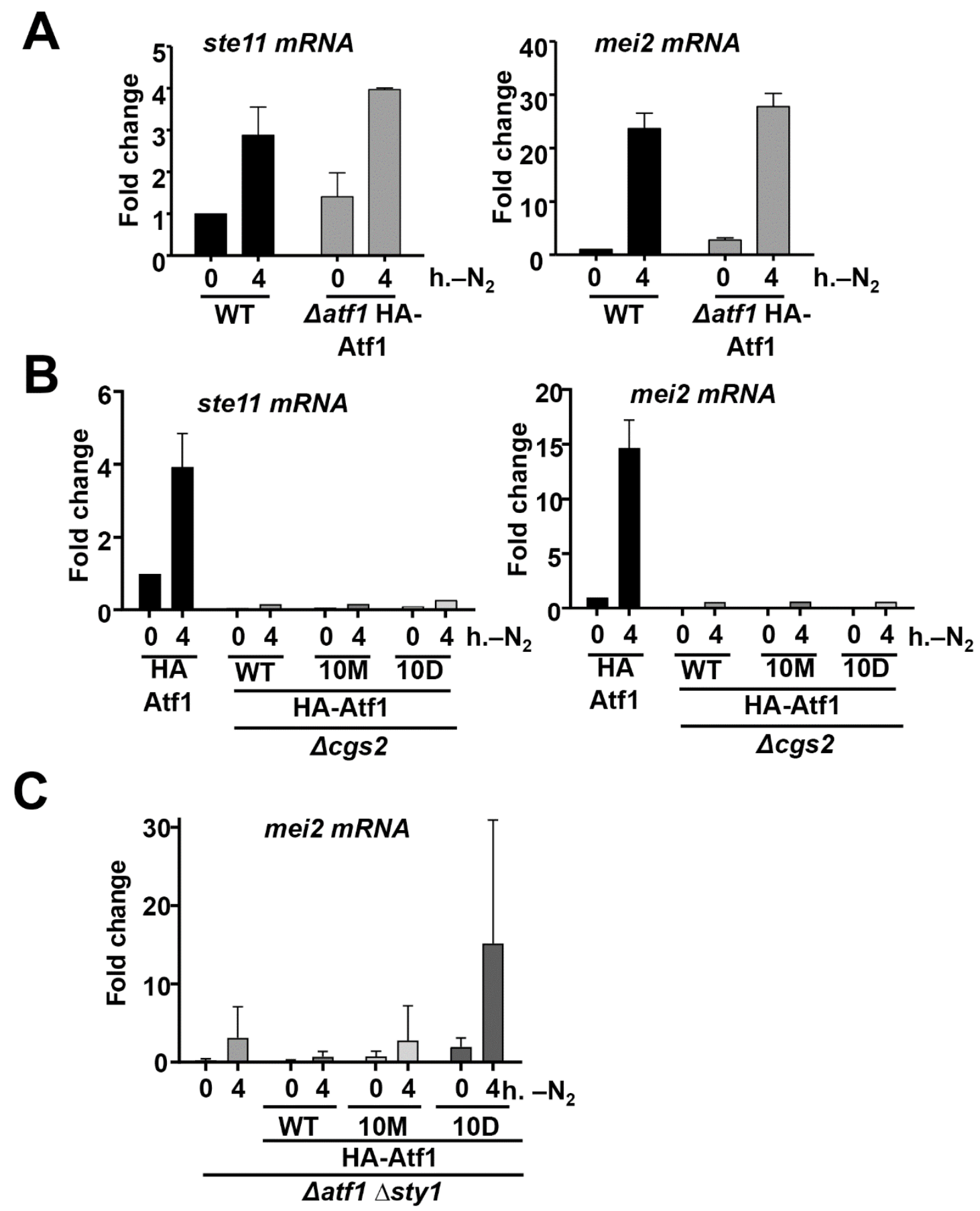

Figure S1. Phosphodiesterase Cgs2 is needed for the regulation of meiosis genes regardless of Atf1 phosphorylation. (A) Samples of the strains 972 (wild type) and EP203 (Datf1 + HA-Atf1) were obtained during exponential growth and 4 hours after switching the culture to low nitrogen media. RNA was extracted and analyzed with ste11 and mei2 primers, and normalized with act1. Fold induction levels were calculated by assigning a value of 1 to the levels in a wild type strain during exponential growth. (B) Expression of ste 11 and mei2 was analyzed as in A, using strains

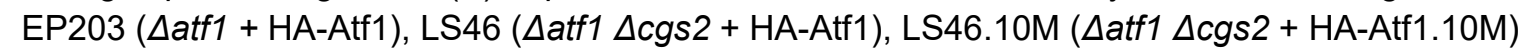
and LS46.10D ( $\Delta$ atf1 $\Delta$ cgs2 + HA-Atf1.10D). (C) Expression of mei2 was analyzed as in A, using strains EP288 ( $\Delta$ sty1 $\Delta$ atf1 + empty plasmid), EP303 ( $\Delta$ sty1 $\Delta$ atf1 + WT HA-Atf1), EP303.10M

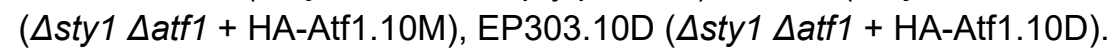



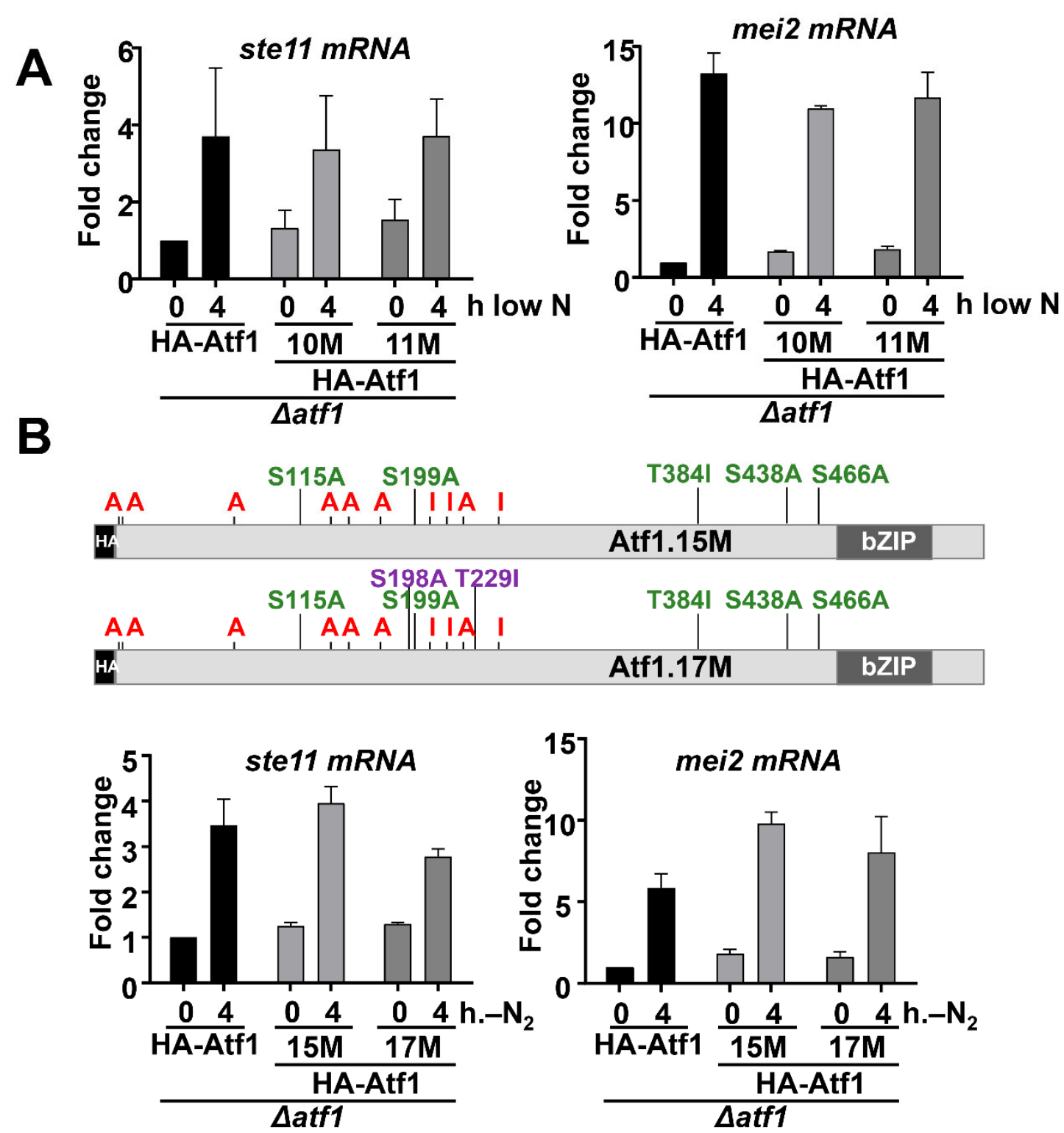

Figure S2. Activation of ste 11 and mei2 is still functional in cells expressing the Atf1.11M, Atf1.15M and 17M mutants. (A) Samples of the strains EP203 ( $\triangle$ atf1 + HA-Atf1), EP203.10M ( $\triangle$ atf1 + HA-Atf1.10M) and EP203.11M ( $\triangle$ atf1 + HA-Atf1.11M) were obtained during exponential growth and 4 hours after switching the cultures to medium lacking nitrogen. RNA was extracted and analyzed with ste11 and mei2 primers, and normalized with act1. Fold induction levels were calculated by assigning a value of 1 to the levels in a wild type strain during exponential growth. (B) Scheme depicting the non-canonical MAPK phosphorylation sites in HA-Atf1.15M and HA-

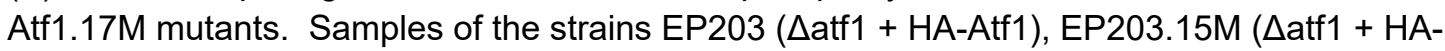
Atf1.15M) and EP203.17M ( $\triangle$ atf1 + HA-Atf1.17M) were obtained during exponential growth and 4 hours after switching the culture to media lacking nitrogen. RNA was extracted and analyzed with ste 11 and mei2 primers, and normalized with act1. Fold induction levels were calculated by assigning a value of 1 to the levels in a wild type strain during exponential growth. 


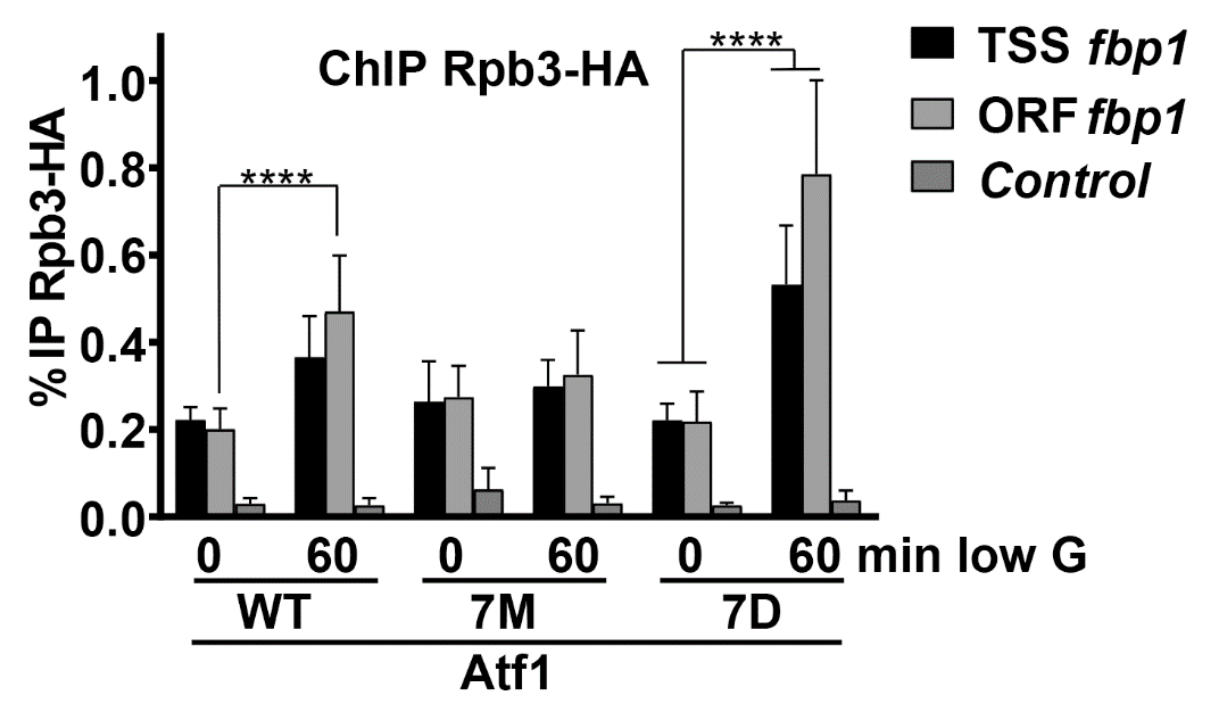

Figure S3. Recruitment of Pol II to fbp1 depends on Atf1 phosphorylation. ChIP experiment using anti-HA antibody to localize Pol II subunit Rpb3 tagged with HA. Samples from strains JF5 (rpb3-HA), CS81.7M (rpb3-HA atf1.7M) and CS81.7D (rpb3-HA atf1.7D) were taken in exponential growth, and 60 minutes after switching the culture to low-glucose media. Data are presented as mean $+/-\mathrm{SD}$; ${ }^{* *}, \mathrm{p}<0.01$; ${ }^{* * * *}, \mathrm{p}<0.0001$ (Student's $t$ test) from biological triplicates. Primers designed to bind act1 ORF were used as control. ( $\triangle$ atf1 + HA-Atf1.10D) were taken during exponential growth and after 60 minutes of glucose deprivation. 

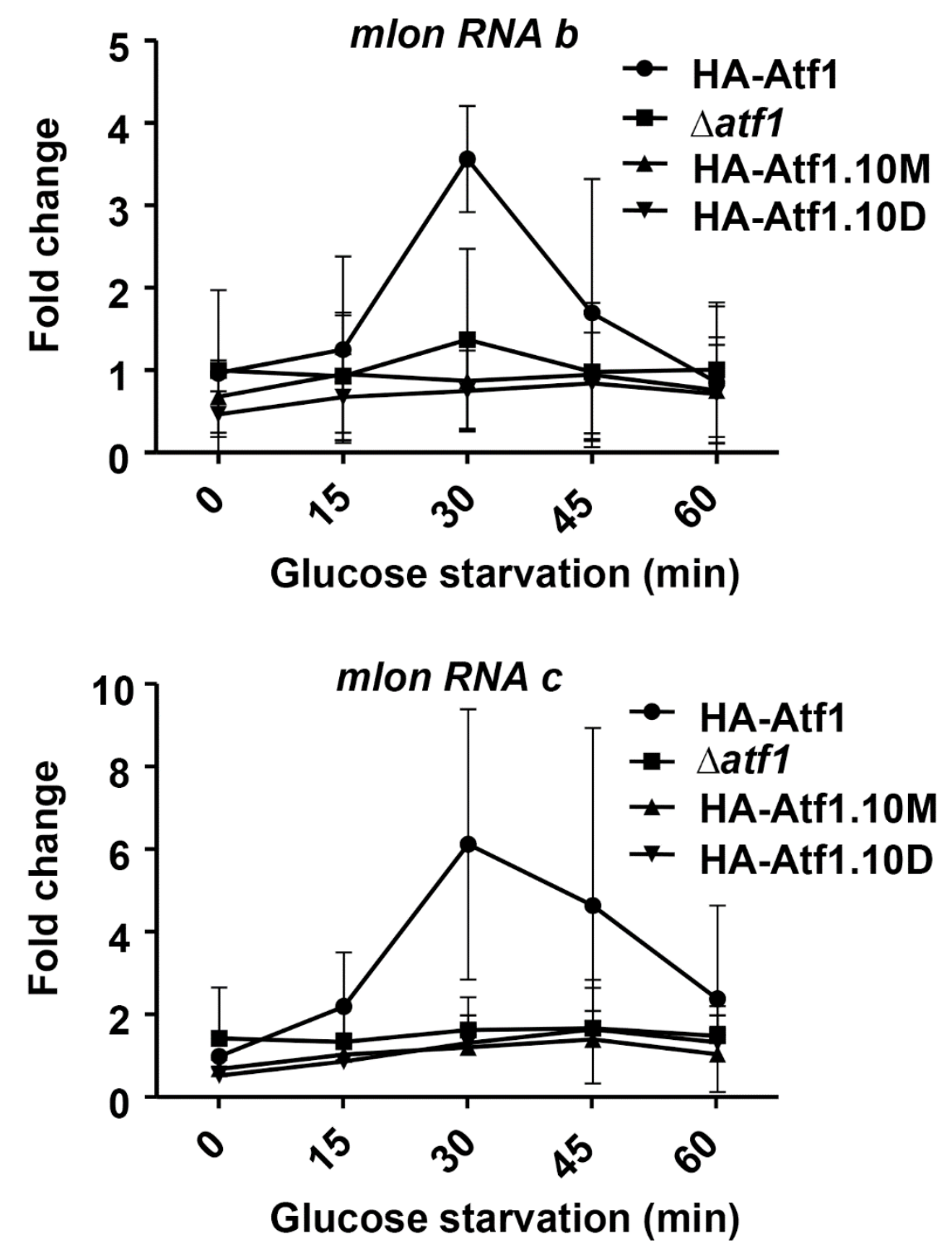

Figure S4. Metabolic long non-coding RNAs (mlonRNA) b and $\mathrm{c}$ transcription rates do not change in phosphomutants Atf1.10M and Atf1.10D. Samples of the strains EP203 ( $\triangle$ atf1 + HA-

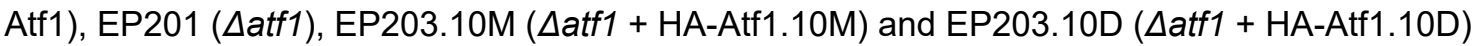
were obtained during exponential growth and every 15 minutes after switching the culture to low glucose media. RNA was extracted and analyzed with specific primers for mlonRNA $b$ (left panel) and $c$ (right panel). Fold induction levels were calculated by assigning a value of 1 to the levels in a wild type strain during exponential growth. 
Table S1. Strains used in this study

\begin{tabular}{|c|c|c|}
\hline Strain & Genotype & Origin \\
\hline 972 & $h^{-}$ & [1] \\
\hline 975 & $h^{+}$ & {$[1]$} \\
\hline EP201 & $h^{-}$atf1::natMX6 sty1'::HA::leu1 leu1-32 & [2] \\
\hline EP203 & $h^{-}$atf1::natMX6 sty1'::HA-atf1::/eu1 leu1-32 & {$[3]$} \\
\hline EP203.10M & $h^{-}$atf1::natMX6 sty1'::HA-atf1.10M::leu1 leu1-32 & {$[2]$} \\
\hline EP203.10D & $h^{-}$atf1::natMX6 sty1'::HA-atf1.10D::leu1 leu1-32 & {$[2]$} \\
\hline EP288 & $h^{-}$atf1::natMX6 sty1::ura4 sty1'::HA::leu1 leu1-32 ura4.D18 & [2] \\
\hline EP303 & $h^{-}$atf1::natMX6 sty1::ura4 sty1'::HA-atf1::leu1 leu1-32 ura4.D18 & [2] \\
\hline EP303.10M & $\begin{array}{l}h^{-} \text {atf1::natMX6 sty1::ura4 sty1'::HA-atf1.10M::leu1 leu1-32 } \\
\text { ura4.D18 }\end{array}$ & [2] \\
\hline EP303.10D & $\begin{array}{l}h^{-} \text {atf1::natMX6 sty1::ura4 sty1'::HA-atf1.10D::leu1 leu1-32 } \\
\text { ura4.D18 }\end{array}$ & {$[2]$} \\
\hline AV18 & $h^{+}$sty $1:: k a n M X 6$ & {$[4]$} \\
\hline EP33 & h-pyp1::natMX6 leu1-32 ura4-D18 & This work \\
\hline EP48 & $h^{-}$pyp1::natMX6 & This work \\
\hline JA123 & $h+$ ade6-M26 leu1-32 & Lab stock \\
\hline JA126 & $h+$ ade6-M375 leu1-32 & Lab stock \\
\hline JA381 & $h$ - ade6-M210 leu1-32 & Lab stock \\
\hline LS9 & $h+$ atf1::natMX6 ade6-M26 leu1-32 & This work \\
\hline LS10 & $h+$ atf1::natMX6 ade6-M375 leu1-32 & This work \\
\hline LS13 & h- atf1::natMX6ade6-M210 leu1-32 & This work \\
\hline LS19 & $h^{+}$atf1::natMX6 ade6-M26 & This work \\
\hline LS20 & $h^{+}$atf1::natMX6 ade6-M375 & This work \\
\hline LS50 & $h^{-}$atf1::natMX6 ade6-M210 & This work \\
\hline LS11 & $h^{+}$atf1::natMX6 sty1'::HA-atf1::leu1+ ade6-M26 leu1-32 & This work \\
\hline LS11.10M & $h^{+}$atf1::natMX6 sty1'::HA-atf1.10M::leu1+ ade6-M26 leu1-32 & This work \\
\hline LS11.10D & $h^{+}$atf1::natMX6 sty1'::HA-atf1.10D::leu1+ ade6-M26 leu1-32 & This work \\
\hline LS12 & $h^{+}$atf1::natMX6 sty1'::HA-atf1::leu1+ ade6-M375 leu1-32 & This work \\
\hline LS12.10M & $h^{+}$atf1::natMX6 sty1'::HA-atf1.10M::leu1+ ade6-M375 leu1-32 & This work \\
\hline LS12.10D & $h^{+}$atf1::natMX6 sty1'::HA-atf1.10D::leu1+ ade6-M375 leu1-32 & This work \\
\hline LS14 & $h^{-}$atf1::natMX6 sty1'::HA-atf1::leu1+ ade6-M210 leu1-32 & This work \\
\hline LS14.10M & $h^{-}$atf1::natMX6 sty1'::HA-atf1.10M::leu1+ ade6-M210 leu1-32 & This work \\
\hline LS14.10D & $h^{-}$atf1::natMX6 sty1'::HA-atf1.10D::leu1+ ade6-M210 leu1-32 & This work \\
\hline LS53 & $\begin{array}{l}h^{+} \text {sty1::ura atf1::natMX6 sty1'::HA::leu1+ ade6-M26 leu1-32 } \\
\text { ura4-D18 }\end{array}$ & This work \\
\hline LS52 & $\begin{array}{l}h^{+} \text {sty1::ura atf1::natMX6 sty1'::HA::leu1+ ade6-M375 leu1-32 } \\
\text { ura4-D18 }\end{array}$ & This work \\
\hline LS59 & $\begin{array}{l}h^{-} \text {sty1::ura atf1::natMX6 sty1'::HA:::leu1+ ade6-M210 leu1-32 } \\
\text { ura4-D18 }\end{array}$ & This work \\
\hline LS24 & \multicolumn{2}{|c|}{$\begin{array}{l}h^{+} \text {sty1::ura atf1::natMX6 sty1'::HA-atf1::leu1+ ade6-M26 leu1-32This work } \\
\text { ura4-D18 }\end{array}$} \\
\hline LS24.10M & $\begin{array}{l}h^{+} \text {sty1::ura atf1::natMX6 sty1'::HA-atf1.10M::leu1+ ade6-M26 } \\
\text { leu1-32 ura4-D18 }\end{array}$ & This work \\
\hline LS24.10D & $\begin{array}{l}h^{+} \text {sty1::ura atf1::natMX6 sty1'::HA-atf1.10D::leu1+ ade6-M26 } \\
\text { leu1-32 ura4-D18 }\end{array}$ & This work \\
\hline LS28 & $\begin{array}{l}h^{+} \text {sty1::ura atf1::natMX6 sty1'::HA-atf1::leu1+ ade6-M375 leu1- } \\
32 \text { ura4-D18 }\end{array}$ & This work \\
\hline
\end{tabular}




\begin{tabular}{|c|c|c|}
\hline LS28.10M & $\begin{array}{l}h^{+} \text {sty1::ura atf1::natMX6 sty1'::HA-atf1.10M::leu1+ ade6-M375 } \\
\text { leu1-32 ura4-D18 }\end{array}$ & This work \\
\hline LS28.10D & $\begin{array}{l}h^{+} \text {sty1::ura atf1::natMX6 sty1'::HA-atf1.10D::leu1+ ade6-M375 } \\
\text { leu1-32 ura4-D18 }\end{array}$ & This work \\
\hline LS56 & $\begin{array}{l}h^{-} \text {sty1::ura atf1::natMX6 sty1'::HA-atf1::leu1+ ade6-M210 leu1- } \\
32 \text { ura4-D18 }\end{array}$ & This work \\
\hline LS56.10M & $\begin{array}{l}h^{-} \text {sty1::ura atf1::natMX6 sty1'::HA-atf1.10M::Ieu1+ ade6-M210 } \\
\text { leu1-32 ura4-D18 }\end{array}$ & This work \\
\hline LS56.10D & $\begin{array}{l}\text { h- sty1::ura atf1::natMX6 sty1'::HA-atf1.10D::leu1+ ade6-M210 } \\
\text { leu1-32 ura4-D18 }\end{array}$ & This work \\
\hline 22G4 & $h^{+}$cgs2::kanMX4 leu1-32 ura4-D18 ade6-M210 & [5] \\
\hline MS110 & $h^{-} \operatorname{cgs} 2:: k a n M X 4$ & This work \\
\hline LS40 & $h^{+}$cgs $2:: k a n M X 4$ & This work \\
\hline $\begin{array}{l}\text { EP193 } \\
\text { LS41 }\end{array}$ & $\begin{array}{l}h^{-} \text {atf1::natMX6 leu1-32 } \\
h+\text { atf1::natMX6 cgs2::kanMX6 leu1-32 }\end{array}$ & $\begin{array}{l}{[2]} \\
\text { This work }\end{array}$ \\
\hline LS46 & $h^{+}$atf1::natMX6 cgs2::kanMX6 sty1'::HA-atf1::leu1 leu1-32 & This work \\
\hline LS46.10M & $h^{+}$atf1::natMX6 cgs2::kanMX6 sty1'::HA-atf1.10M::leu1 leu1-32 & This work \\
\hline LS46.10D & $h^{+}$atf1::natMX6 cgs2::kanMX6 sty1'::HA-atf1.10D::leu1 leu1-32 & This work \\
\hline LS106 & $h^{-}$atf1::natMX6 sty1'::HA-atf1::leu1 rst2-FLAG::kanMX6 leu1-32 & This work \\
\hline LS106.10M & $\begin{array}{l}h^{-} \text {atf1::natMX6 sty1'::HA-atf1.10M::leu1 rst2-FLAG::kanMX6 } \\
\text { leu1-32 }\end{array}$ & This work \\
\hline LS106.10D & $\begin{array}{l}h^{-} \text {atf1::natMX6 sty1'::HA-atf1.10D::leu1 rst2-FLAG::kanMX6 } \\
\text { leu1-32 }\end{array}$ & This work \\
\hline LS116 & $h^{-}$atf1::natMX6 sty1'::HA::Ieu1 rst2-FLAG::kanMX6 leu1-32 & This work \\
\hline JX233 & h90 rst2::ura4 ade6-M216 leu1 ura4-D18 & [6] \\
\hline LS85 & h- atf1::natMX6 rst2::ura4 leu1-32 ura4.D18 & This work \\
\hline RF22 & $h^{-}$atf1::natMX6 rst2::ura4 sty1'::HA-atf1::leu1 leu1-32 ura4.D18 & This work \\
\hline RF22.10M & $\begin{array}{l}h^{-} \text {atf1::natMX6 rst2::ura4 sty1'::HA-atf1.10M::leu1 leu1-32 } \\
\text { ura4.D18 }\end{array}$ & This work \\
\hline RF22.10D & $\begin{array}{l}h^{-} \text {atf1::natMX6 rst2::ura4 sty1'::HA-atf1.10D::leu1 leu1-32 } \\
\text { ura4.D18 }\end{array}$ & This work \\
\hline RF94 & $h^{-}$atf1::natMX6 pka1::kanMX6 sty1'::HA-atf1::leu1 leu1-32 & This work \\
\hline RF95 & $h^{-}$atf1::natMX6 pka1::kanMX6 sty1'::HA:::leu1 leu1-32 & This work \\
\hline 25B1 & $h^{+}$pka1::kanMX4 leu1-32 ura4-D18 ade6-M210 & [5] \\
\hline AZ75 & pka1::kanMX6 leu1-32 & This work \\
\hline AZ61 & pka1::kanMX6 sty1::ura & This work \\
\hline LS105 & $h^{-}$atf1::natMX6 scr1::kanMX6 sty1'::HA-atf1::leu1 leu1-32 & This work \\
\hline RF103.10D & h- atf1::natMX6 scr1::kanMX6 sty1'::HA-atf1.10D::leu1 leu1-32 & This work \\
\hline JF5 & $h^{-}$rpb3-HA::natMX6 & (3) \\
\hline CS81.7M & $h^{-}$rpb3-HA::natMX6 atf1.7M & (3) \\
\hline CS81.7D & $h^{-}$rpb3-HA::natMX6 atf1.7D & (3) \\
\hline
\end{tabular}


Table S2. Primers used in this study for RT-qPCR, ChIP and nucleosome scanning experiments

\begin{tabular}{|c|c|c|c|}
\hline Primer & Sequence & Gene $^{a}$ & Application \\
\hline OLEH-N7 & 5'-ACACTAGACGAAGAGACGCATGTAT-3' & ste11 $\mathrm{F}$ & RT-qPCR \\
\hline OLEH-N8 & 5'-ACCGAACTTTTATCTACGCCTTATT-3' & ste11 R & RT-qPCR \\
\hline OLEH-N9 & 5'-TTTGTAAGCCATGGACCTAATAGA-3' & mei2 F & RT-qPCR \\
\hline OLEH-N10 & 5'-TATTTGTTGGGCACTAGCAATAGAT-3' & mei2 $\mathrm{R}$ & RT-qPCR \\
\hline OLEH-S41 & 5'-AAAACGTACAACACTATACCAAAGCA-3' & mlon RNA $b$ F & RT-qPCR \\
\hline OLEH-S42 & 5'-GTATGATTGATCGCCATTGTGTAG-3' & mlon RNA $b \mathrm{R}$ & RT-qPCR \\
\hline OLEH-S43 & 5'-AGTCACGTAAACCTCGATACGATAC-3' & mlon RNA $c$ F & RT-qPCR \\
\hline OLEH-S44 & 5'-GАСTCАTTCСАСССТАТTСАТСТTT-3' & mlon RNA $c$ R & RT-qPCR \\
\hline OLEH-S77 & 5'-AAAACCATACTCTGCTCGATACATT-3' & ORF fbp $1 \mathrm{~F}$ & RT-qPCR/ ChIP \\
\hline OLEH-S78 & 5'-TTATTTCCTTTTGAGCATGGATAAG-3' & ORF fbp1 R & RT-qPCR/ ChIP \\
\hline OLEH-H48 & 5'-TGCCCCTAGAGCTGTATTCC-3' & $\operatorname{act1} F$ & RT-qPCR/ ChIP \\
\hline OLEH-H49 & 5'-TTTGAGCTTCATCACCAACG-3'- & act1 $R$ & RT-qPCR/ ChIP \\
\hline OLEH-R76 & 5'-СТААСТАСТССАТССАСССА-3' & UAS1 fbp1 F & ChIP \\
\hline OLEH-R77 & 5'-AAGCAAAATCAGAAATTGTG-3' & UAS1 fbp1 R & ChIP \\
\hline OLEH-R78 & 5'-TGTTTAGCAGGCTGAAACAG-3' & UAS2 fbp1 F & ChIP \\
\hline OLEH-R79 & 5'-CCGCTTAATTAAAAATGCAT-3' & UAS2 fbp1 R & ChIP \\
\hline OLEH-S45 & 5'-GTTGTTTACATGGGAAATACATGG-3' & TSS fbp1 F & ChIP \\
\hline OLEH-S46 & 5'-CTAACAAACGTTTCACCTTGACTG-3' & TSS fbp1 R & ChIP \\
\hline OLEH-T69 & 5'-TCССTCTCTTTAATTACCTGGATGAC-3' & fbp1 $-1307 b p$ & Nucl. Scann. \\
\hline OLEH-T70 & 5'-AGTAGTTAGTAGGAGGAGAAATGAACC-3' & fbp $1-1205 b p$ & Nucl. Scann. \\
\hline OLEH-T71 & 5'-ТСТССТССТАСТААСТАСТССАТСС-3' & $f b p 1-1224 b p$ & Nucl. Scann. \\
\hline OLEH-T72 & 5'-CAGAAATTGTGAAATTCGATGATGAGT-3' & $f b p 1-1122 b p$ & Nucl. Scann. \\
\hline OLEH-T73 & 5'-CGAATTTCACAATTTCTGATTTTCG-3' & fbp1-1140 bp & Nucl. Scann. \\
\hline OLEH-T74 & 5'-CCTGTGTTAACTCAGAATCAACATA-3' & fbp1-1039 bp & Nucl. Scann. \\
\hline OLEH-T75 & 5'-AGCCATGTTATGTTGATTCTGAGTT-3' & fbp1 $1-1072 b p$ & Nucl. Scann. \\
\hline OLEH-T76 & 5'-TAGTGTTGTACGTTTTAGAGCACAC-3' & fbp1 -971 bp & Nucl. Scann. \\
\hline OLEH-T79 & 5'-TACACAATGGCGATCAATCATACAC-3' & fbp1-912 bp & Nucl. Scann. \\
\hline OLEH-T80 & 5'-ACCGACCTCAATTGCATACCCACAG-3' & $f b p 1-812 b p$ & Nucl. Scann. \\
\hline OLEH-T81 & 5'-TCGATCTTATGTAATTGCTGTGGGT-3' & fbp $1-854 b p$ & Nucl. Scann. \\
\hline OLEH-T82 & 5'-TGGCACTCCCTTAATACATTCGTAA-3' & fbp $1-752 b p$ & Nucl. Scann. \\
\hline OLEH-T83 & 5'-TTTACGAATGTATTAAGGGAGTGCC-3' & fbp $1-778$ bp & Nucl. Scann. \\
\hline OLEH-T84 & 5'-GTAAATACGGGATGGGAAGGTTAGA-3' & $f b p 1-676 b p$ & Nucl. Scann. \\
\hline OLEH-T85 & 5'-ATACAAGCTCTAАССТTCССАТCCC-3' & fbp $1-709$ bp & Nucl. Scann. \\
\hline OLEH-T86 & 5'-GGCTTACCAAACGATTGCTAGAGCG-3' & $f b p 1-608 b p$ & Nucl. Scann. \\
\hline OLEH-T87 & 5'-GAGTCCGCTCTAGCAATCGTTTGGT-3' & $f b p 1-637 b p$ & Nucl. Scann. \\
\hline OLEH-T88 & 5'-CTTGCAATGAAAACTACAGGGCAAT-3' & $f b p 1-534 b p$ & Nucl. Scann. \\
\hline OLEH-T89 & 5'-TGCCCTGTAGTTTTCATTGCAAG-3' & fbp $1-557 b p$ & Nucl. Scann. \\
\hline OLEH-T90 & 5'-CTATTTCATACGCCCACCTGAGT-3' & fbp $1-455 b p$ & Nucl. Scann. \\
\hline OLEH-T91 & 5'-ACTCAGGTGGGCGTATGAAATAG-3' & fbp1-478bp & Nucl. Scann. \\
\hline OLEH-T92 & 5'-GCTATGTTTAGTTCCGCGAATCATA-3' & fbp1-380bp & Nucl. Scann. \\
\hline OLEH-T93 & 5'-TATGATTCGCGGAACTAAACATAGCG-3' & $f b p 1-405 b p$ & Nucl. Scann. \\
\hline OLEH-T94 & 5'-ACAAAAATCAACGAGCCATGATGGA-3' & fbp1 -303 bp & Nucl. Scann. \\
\hline
\end{tabular}




\begin{tabular}{|c|c|c|c|}
\hline OLEH-T95 & 5'-TCCATCATGGCTCGTTGATTTTTGT-3' & fbp $1-328$ bp & Nucl. Scann. \\
\hline OLEH-T96 & 5'-GTCTCTCTCCAGTGTAAACAAAA-3' & $f b p 1-226 b p$ & Nucl. Scann. \\
\hline OLEH-V29 & 5'-ACTGGAGAGAGACAACACAACTC-3' & fbp $1-239$ bp & Nucl. Scann. \\
\hline OLEH-V30 & 5'-AAGTTGGGCTAGAAACCGAGTG-3' & fbp1 1 -138 bp & Nucl. Scann. \\
\hline OLEH-V31 & 5'-CCACTCGGTTTCTAGCCCAACTTAA-3' & fbp1 $1-160$ bp & Nucl. Scann. \\
\hline OLEH-V32 & 5'- GGGGAATGAAGTTGAATAAGCTGGC-3' & $f b p 1-59 b p$ & Nucl. Scann. \\
\hline
\end{tabular}

aWhen included, the number indicates the position of the 5' end of the primer relative to the translation start point

\section{SUPPLEMENTAL REFERENCES}

[1] Leupold U. Genetical methods for Schizosaccharomyces pombe. Methods Cell Physiol. 1970;4:169-77.

[2] Salat-Canela C, Paulo E, Sanchez-Mir L, Carmona M, Ayte J, Oliva B, et al. Deciphering the role of the signal- and Sty1 kinase-dependent phosphorylation of the stress-responsive transcription factor Atf1 on gene activation. J Biol Chem. 2017;292:13635-44.

[3] Garcia P, Paulo E, Gao J, Wahls WP, Ayte J, Lowy E, et al. Binding of the transcription factor Atf1 to promoters serves as a barrier to phase nucleosome arrays and avoid cryptic transcription. Nucleic Acids Res. 2014;42:10351-9.

[4] Zuin A, Vivancos AP, Sanso M, Takatsume Y, Ayte J, Inoue Y, et al. The glycolytic metabolite methylglyoxal activates Pap1 and Sty1 stress responses in Schizosaccharomyces pombe. J Biol Chem. 2005;280:36708-13.

[5] Roguev A, Wiren M, Weissman JS, Krogan NJ. High-throughput genetic interaction mapping in the fission yeast Schizosaccharomyces pombe. Nat Methods. 2007;4:861-6.

[6] Higuchi T, Watanabe Y, Yamamoto M. Protein kinase A regulates sexual development and gluconeogenesis through phosphorylation of the $\mathrm{Zn}$ finger transcriptional activator Rst2p in fission yeast. MolCell Biol. 2002;22:1-11. 